\title{
A stochastic model to evaluate pricing distortions in indemnity insurance methods for MTPL insurance
}

\author{
Paola Fersini $^{1}$ D $\cdot$ Salvatore Forte ${ }^{2} \cdot$ Giuseppe Melisi $^{3} \cdot$ Gennaro Olivieri $^{1}$
}

Received: 8 August 2018 / Accepted: 1 March 2019 / Published online: 27 March 2019

(C) The Author(s) 2019

\begin{abstract}
Direct compensation or the direct reimbursement scheme is an indemnity insurance method that many European and American countries use to manage motor liability claims in which the driver that suffers an accident is paid by his/her insurance company that possibly later receives a flat-rate reimbursement (known as forfeit). Using non-life actuarial methodologies, this article analyses the distortion effects due to the direct compensation mechanisms and the effects of different forfeit reimbursement systems on policyholder tariffs in the management of motor liability claims involving vehicles in two different sectors, i.e. automobile and motorcycle. We empirically analyse and formalize the distortion effects resulting from the mechanism that different direct reimbursement systems produce, and explore the correlation between increasing tariffs for motorcycle policyholders and decreasing tariffs for other vehicle policyholders. We propose some alternative methods to overcome these distortion effects, evaluating their pricing impact through a stochastic model applied to a case study.
\end{abstract}

Keywords Direct compensation · Pricing distortions - Motor third party liability · Pure premium $\cdot$ Simulation

Mathematics Subject Classification 62P05 $\cdot 91 \mathrm{~B} 30 \cdot 65 \mathrm{C} 05$

JEL Classification G22

\section{Introduction}

The auto insurance market has received extensive attention in research as it represents about $40 \%$ of all property and casualty insurance net premiums in the USA (NAIC 2016) and the largest non-life business line in Europe in 2015, accounting for

Paola Fersini

pfersini@1uiss.it

Extended author information available on the last page of the article 
$38 \%$ of total non-life premiums (Insurance Europe 2016). The first relevant research stream addresses the analysis of the effect of the liability rule, comparing two compensation systems: tort law (typical of European countries) and pure no-fault (typical of Australia, New Zealand, and Canada), and the factors that influence the formation of motor prices including the effects of rate regulation on the automotive insurance market (e.g. Grabowski et al. 1989; Cummins and Tennyson 1992; Bouzouita and Bajtelsmit 1997; Tennyson 1997; Yu-Luen and Schmit 2000; Kelly et al. 2010; Grace and Leverty 2012; Born 2017). Other authors investigate the relations between the incidence of uninsured drivers and the price of coverage (Dahlby 1983; Gastel 1998; Sugarman 1998). Numerous authors exhaustively examine the characteristics of automotive insurance markets in Canada, in Europe (Schmitt 2000; Cummins 2002; Cuocci 2013), and in the USA (Graham and Xie 2007; Cuocci 2013), and the various factors affecting tariffs, including deregulation (the change of markets from a cartel pricing situation to complete pricing freedom; see Cummins 2007), distribution system, rules governing statutory reporting and local accounting standards, Bonus/Malus schemes, legal system, taxes, policy cover (first party, third party, etc.). The analysis is more complex in the USA, where the insurance discipline is subject to state legislation. Some states favour a no-fault system; others maintain a civil liability system. However, states that favour a no-fault system do not have a uniform system that distinguishes between threshold no-fault, add no-fault, and choice no-fault. Each of these no-fault schemes preserves third party liability in some way. Joost (2002) and Schermer and Schermer (2004) provide a complete examination of motor vehicle damage insurance in the USA. The second research stream concerns the specific literature on direct compensation, including studies that examine the functioning of different types of direct compensation and their implications in terms of overall system costs and distribution between companies (Galli and Savino 2007; De Santis 2006), and studies analysing the influence of direct compensation on determining technical reserves (Fersini and Melisi 2016).

Traditionally, for motor vehicle damage, policyholders must request payment for damages from the other party. However, in some countries (e.g. France, Italy, Portugal, Spain, Canada, the USA), agreements exist between insurers concerning direct reimbursement of certain types of claims. In such schemes, in case of road accident claims, the insurance company of the injured party reimburses the damage to its policyholder. In Canada (Quebec, Ontario, New Brunswick), for example, a public monopoly offers compensation for bodily injuries in a pure no-fault system, and a competitive private sector offers compensation for property damage under a liability system that includes a direct compensation agreement designed to reduce insurance costs. In 1978, private insurers began awarding direct compensation to victims of property damage according to the Direct Compensation Agreement between insurers. This is called "direct compensation" because, even though someone else caused the damage, the insured person collects from his/her insurer directly instead of from the person who caused the accident. In Italy, after the introduction of the direct compensation system (CARD), ${ }^{1}$ the driver that suffers an accident is paid by his/her

\footnotetext{
${ }^{1}$ Direct compensation (CARD) was introduced in Italy by Decree-Law n. 254/06.
} 
own insurance company. Subsequently the insurance company will receive a reimbursement forfeit from the insurance company of the driver responsible for the accident. However, the insurance company of the responsible party will not reimburse the victim's insurance company the exact sum that the victim received from his/her own insurance company, but as stated, a forfeit reimbursement. In Italy, these forfeit reimbursements are defined year by year based of some parameters determined by CONSAP (the insurance services entity that manages the reimbursement system between insurance companies). Until 2007, before the introduction of the CARD system, the driver that incurred a claim was paid by the insurance company of the driver that caused the claim. In general, direct reimbursement (DR) schemes maintain the principle of responsibility with the payment made by those causing the accident. The object of insurance coverage, irrespective of the agreements between the insurance companies on the payment mechanism, remains liability with respect to third parties or the property of third parties. The actuarial methodologies are the same, with appropriate integrations, and the insured pay a premium in line with this specific risk.

This article looks at the effect of various forms of insurance claim handling on policyholder tariffs, focusing on the direct indemnity mechanism when two different types of vehicles are involved (i.e. automobile and motorcycle; motorcycle and truck; automobile and truck; motorcycle and truck; etc.). We analyse different direct compensation schemes where the forfeit payment is made by the company of the insured who caused the claim, comparing the results with traditional payment mechanism without direct compensation and investigating the distortions created in the price coverage in the various cases examined. In the present context, price distortions are defined as deviations of insurance prices from a level that should be applied in the market if insurance companies charge tariffs for each business line that correspond, on average, to the accident caused by the vehicles of the same business line. Price distortions lead to mispricing due to the mechanism inherent in different direct reimbursement systems.

We then propose some solutions that could reduce or eliminate this distortion effect. First, we formalize and determine a market premium for the different schemes; second, we analyse a market distortion index. In conclusion, through a simulation, we present a case study based on realistic data from three Italian companies using a stochastic model. In particular, the data refer to three Italian companies selected to cover the small, medium, and large size classes. Furthermore, due to privacy issues, the data have been slightly modified to avoid the potential identification of the companies that asked to remain anonymous.

Finally, we do not aim to investigate what has occurred in the Italian market (previously analysed in Fersini et al. 2017), but the distorting effects of the various existing and potential DR schemes on pricing. Therefore, the hypothesis of a system consisting of only three companies does not compromise the validity of the results obtained and the final considerations.

The structure of this paper is as follows: Sect. 2 briefly specifies the traditional reimbursement scheme for MTPL. Section 3 describes three different types of direct claim settlements to policyholders (the first of which corresponds to that in force in Italy) and illustrates the calculation for each business line of pure premium, market 
premium, and a type of solidarity market index. Thereafter, an alternative direct reimbursement method with forfeit is presented in which the total costs of each sector can depend exclusively on the costs of third party liability of the same business line. Section 4 develops the simulation-based framework that enables determining the distortion caused by the introduction of the direct compensation mechanism with forfeit adjustment and illustrates the empirical application and model comparison. The last section concludes the paper.

\section{The traditional reimbursement scheme: modelling framework}

Motor third party liability (MTPL), compulsory in the EU, provides financial protection against claims for physical damage and/or bodily injury resulting from traffic collisions. Additionally, comprehensive motor insurance offers financial protection for first party losses and, in some cases, fire, theft, and breakdown services. The traditional reimbursement scheme for MTPL, without a direct compensation system, can be formalized in the following way.

For the sake of simplicity, we assume that in the insurance market, there are $n$ MTPL insurance companies that insure vehicles belonging to $q$ different sectors. We indicate with $a_{i}^{l}$, with $i=1,2, \ldots . n$ insurance companies and $l=1,2, \ldots, q$ sectors, the expected number of vehicles that will be insured in the reference period by $n$ insurance companies for each $q$ sector.

Then, we indicate with $k(i, j)^{l, m}$ the expected claim number caused by owners/ drivers of vehicles of sector $l$ insured by company $i$ against owners/drivers of vehicles of sector $m$ insured by company $j$. With $i=1,2, \ldots, n ; j=1,2, \ldots, n$ and $i \neq j$; $l=1,2, \ldots, q ; m=1,2, \ldots, q$.

The limitation that $i$ must differ from $j(i \neq j)$ implies that there are no claims belonging to the same sector or to different sectors insured by the same company (so-called natural claims). To be noted is that these claims are not numerically consistent in the real insurance market and hence do not condition the results obtained in this work. Furthermore, given our objective, including these claims within the model has no effect on the market distortion result that depends exclusively on the differences between the sectors and not between the companies. In fact, this typology of claims is excluded from the forfeit mechanism, the CARD system (which we will explain later).

We indicate with:

$$
s_{i}^{l}=\sum_{j=1 ; j \neq i}^{n} \sum_{m=1}^{q} k(i, j)^{l, m} \quad i=1,2, \ldots, n ; l=1,2, \ldots, q
$$

the expected number of claims for which the owners/drivers of vehicles of sector $l$ insured by company $i$ are responsible (all in the same period of reference).

We indicate further with $c(i, j)^{l, m}$ the expected cost of claims caused by the owners/drivers of vehicles of sector $l$ insured by company $i$ and by the owners/drivers of 
vehicles of sector $m$ insured by company $j$. With $i=1,2, \ldots, n ; j=1,2, \ldots, n ; i \neq j$; $l=1,2, \ldots, q ; m=1,2, \ldots, q$.

Finally, we indicate with

$$
c_{i}^{l}=\frac{\sum_{j=1 ; j \neq i}^{n} \sum_{m=1}^{q} k(i, j)^{l, m} * c(i, j)^{l, m}}{s_{i}^{l}} \quad i=1,2, \ldots, n ; l=1,2, \ldots, q
$$

the expected cost of claims for which the owners/drivers of vehicles of sector $l$ insured by company $i$ are responsible (all in the same period of reference).

With these notations, the pure premiums (in this paper with pure premium (or equivalence premium) we indicate the expected value of the aggregate claims without considering the implicit loading or the expected profits for the insurer) that must be paid for the vehicles insured during the reference period for each of the $n$ insurance companies and for each of the $q$ sectors will be:

$$
\pi_{i}^{l}=\frac{s_{i}^{l} * c_{i}^{l}}{a_{i}^{l}} \quad i=1,2, \ldots, n ; l=1,2, \ldots, q
$$

The pure market premiums for the $q$ sectors will be:

$$
\pi_{M}^{l}=\frac{\sum_{i=1}^{n} \sum_{j=1 ; j \neq i}^{n} \sum_{m=1}^{q} k(i, j)^{l, m} * c(i, j)^{l, m}}{\sum_{i=1}^{n} a_{i}^{l}} \quad \text { with } l=1,2, \ldots, q
$$

\section{Direct reimbursement schemes: effects on the premiums}

In this section, we first introduce the framework of the direct reimbursement scheme actually used in Italy and then present the description of other types of direct reimbursements.

\subsection{The current direct reimbursement scheme in Italy}

In the Italian CARD system, the driver that suffers an accident is paid by his/her own insurance company. Subsequently, the insurance company will receive a forfeit reimbursement from the insurance company of the driver responsible for the accident. However, the insurance company of the responsible party will not reimburse the victim's insurance company the exact sum that the victim received from his/her own insurance company, but as stated, a forfeit reimbursement. In Italy, these forfeit reimbursements are defined year by year based of some parameters determined by CONSAP (the insurance services entity that manages the reimbursement system between insurance companies). Until 2007, before the introduction of the CARD system, the driver that incurred a claim was paid by the insurance company of the driver that caused the claim. In general, direct reimbursement (DR) schemes maintain the principle of responsibility with the payment made by those causing the accident. The object of insurance coverage, irrespective of the agreements between the insurance companies on the payment 
mechanism, remains liability with respect to third parties or the property of third parties. The actuarial methodologies are the same, with appropriate integrations, and the insured pay a premium in line with this specific risk.

Relevant here is the distribution of the number of claims of owners/drivers of vehicles insured by each insurance company that will be paid by the other insurance companies according to the type of vehicle involved and the average costs. In this article, we assume that average costs do not vary in the scheme without direct reimbursement and in the DR scheme, in other words, the average costs are independent of who pays. In reality, there are also claims that are not included in the CARD system, but we do not consider these in this paper.

With the data and information available, it is possible to quantify the forfeit for each $q$ sector (calculated in such a way that they result only in the average cost of payments made by insurance companies to own victims):

$$
f_{m}=\frac{\sum_{l=1}^{q} \sum_{i=1}^{n} \sum_{j=1 ; j \neq i}^{n} k(i, j)^{l, m} * c(i, j)^{l, m}}{\sum_{l=1}^{q} \sum_{i=1}^{n} \sum_{j=1 ; j \neq i}^{n} k(i, j)^{l, m}} \quad m=1,2, \ldots, q
$$

It follows that the insurance company of the victim receives a reimbursement that may be higher or lower than the claim amount actually paid to the insured, and can thus make a profit or a loss on the claim of its own insured (the victim without fault). This necessarily affects the pricing that the insurance company will apply to its policyholders. All the insurance companies:

a. pay, with a forfeit differing by business line, the insurance company of the victims of their insured responsible for the claims;

b. pay the claims of their insured (victims, non-responsible claimants);

c. receive the forfeit, differing by business line, from the insurance company of those responsible for the claims that have caused damage to their insured.

If we assume that the expected values are realistic and that the expected forfeits are equal to those that should be paid or received from the insurance companies (in reality, there is a 1-year lag between setting the forfeits and their use in payments/receivables), then we can quantify the premium that the insurance companies will ask of their insured for each $q$ business line so that each insurance company and the insurance market will be in equilibrium.

We indicate with CDR the current direct reimbursement

$$
\begin{aligned}
\pi(\mathrm{CDR})_{j}^{m}= & \frac{\left\{\sum_{p=1}^{q} \sum_{i=1 ; i \neq j}^{n} k(i, j)^{p, m} * c(i, j)^{p, m}-f_{m} *\left[\sum_{p=1}^{q} \sum_{i=1 ; i \neq j}^{n} k(j, i)^{p, m}-\sum_{p=1}^{q} \sum_{i=1 ; i \neq j}^{n} k(i, j)^{p, m}\right]\right\}}{a_{j}^{m}} \\
& \text { with } j=1,2, \ldots, n \text { and } m=1,2, \ldots, q
\end{aligned}
$$

and the pure market premiums for each $q$ business line, with the actual direct reimbursement: 


$$
\begin{aligned}
\pi(\mathrm{CDR})_{M}^{m}= & \frac{\left[\sum_{l=1}^{q} \sum_{i=1}^{n} \sum_{j=1 ; j \neq i}^{n} k(i, j)^{l, m}\right] * f_{m}}{\sum_{j=1}^{n} a_{j}^{m}} \\
= & \frac{\sum_{l=1}^{q} \sum_{i=1}^{n} \sum_{j=1 ; j ; i}^{n} k(i, j)^{l, m} * c(i, j)^{l, m}}{\sum_{j=1}^{n} a_{j}^{m}} \\
= & \pi_{M}^{m}+\frac{\left[\sum_{l=1 ;: \neq m}^{q} \sum_{i=1}^{n} \sum_{j=1 ; j \neq i}^{n} k(i, j)^{l, m} * c(i, j)^{l, m}-\sum_{l=1 ; l \neq m}^{q} \sum_{i=1}^{n} \sum_{j=1 ; j \neq i}^{n} k(i, j)^{m, l} * c(i, j)^{m, l}\right]}{\sum_{j=1}^{n} a_{j}^{m}} \\
& \text { with } m=1,2, \ldots, q
\end{aligned}
$$

It is also possible to define the percentage variation in the market premium relative to the business line following the introduction of the direct reimbursement system:

$$
\begin{aligned}
\Delta^{m}= & \frac{\left[\sum_{l=1 ; l \neq m}^{q} \sum_{i=1}^{n} \sum_{j=1 ; j \neq i}^{n} k(i, j)^{l, m} * c(i, j)^{l, m}-\sum_{l=1 ; l \neq m}^{q} \sum_{i=1}^{n} \sum_{j=1 ; j \neq i}^{n} k(i, j)^{m, l} * c(i, j)^{m, l}\right]}{\sum_{j=1}^{n} a_{j}^{m} * \pi_{j}^{m}} \\
= & \frac{\pi(\mathrm{CDR})_{M}^{m}-\pi_{M}^{m}}{\pi_{M}^{m}} \quad \text { with } m=1,2, \ldots, q
\end{aligned}
$$

The amount $\left[\sum_{l=1 ; l \neq m}^{q} \sum_{i=1}^{n} \sum_{j=1 ; j \neq i}^{n} k(i, j)^{l, m} * c(i, j)^{l, m}-\sum_{l=1 ; l \neq m}^{q} \sum_{i=1}^{n} \sum_{j=1 ; j \neq i}^{n} k(i, j)^{m, l} * c(i, j)^{m, l}\right]$ represents the sum of the "solidarity" (cross-subsidization) of the $m^{\circ}$ business line expressed in terms of premiums.

In other words, it represents the quantity of premiums (in absolute terms) that are paid more (if positive) or less (if negative) for the $m^{\circ}$ business line with the introduction of direct reimbursement compared to without DR.

Furthermore, $\pi(\mathrm{CDR})_{M}^{m}$ is equal to $\pi_{M}^{m}$ for each $m=1,2, \ldots, q$, in the given hypothesis only in the case in which:

$$
\sum_{l=1 ; l \neq m}^{q} \sum_{i=1}^{n} \sum_{j=1 ; j \neq i}^{n} k(i, j)^{l, m} * c(i, j)^{l, m}=\sum_{l=1 ; l \neq m}^{q} \sum_{i=1}^{n} \sum_{j=1 ; j \neq i}^{n} k(i, j)^{m, l} * c(i, j)^{m, l}
$$

Thus, when limited to only two business lines, which we call I and V, the aggregate claim costs sustained by all insurance companies for reimbursements paid to the insured of business line $\mathrm{V}$ caused by business line I are equal to the aggregate claim costs sustained by all insurance companies for reimbursements paid to the insured of business line I caused by business line V. This is practically impossible when the vehicles involved belong to two different business lines.

The generalization to $q$ business lines is quite simple and can also be defined as a market solidarity index with the following formula: 


$$
\begin{aligned}
I_{M} & =\frac{\sum_{m=1}^{q}\left|\left[\sum_{l=1 ; l \neq m}^{q} \sum_{i=1}^{n} \sum_{j=1 ; j \neq i}^{n} k(i, j)^{l, m} * c(i, j)^{l, m}-\sum_{l=1 ; l \neq m}^{q} \sum_{i=1}^{n} \sum_{j=1 ; j \neq i}^{n} k(i, j)^{m, l} * c(i, j)^{m, l}\right]\right|}{\sum_{m=1}^{q} \sum_{j=1}^{n} a_{j}^{m} * \pi_{j}^{m}} \\
& =\frac{\sum_{m=1}^{q}\left|\left[\sum_{l=1 ; l \neq m}^{q} \sum_{i=1}^{n} \sum_{j=1 ; j \neq i}^{n} k(i, j)^{l, m} * c(i, j)^{l, m}-\sum_{l=1 ; l \neq m}^{q} \sum_{i=1}^{n} \sum_{j=1 ; j \neq i}^{n} k(i, j)^{m, l} * c(i, j)^{m, l}\right]\right|}{\sum_{m=1}^{q} \sum_{j=1}^{n} a_{j}^{m} * \pi(\mathrm{CDR})_{j}^{m}}
\end{aligned}
$$

This represents, in percentage terms, the overall variation (in absolute value) in the premiums due to the introduction of CDR with respect to without DR.

Since the balance of these movements influences the tariffs that will be paid by own policyholders and will be compared with the tariffs of other insurance companies on the market, the direct reimbursement mechanism tends to render the system efficient as a whole (with the "solidarity" component between sectors highlighted above) and in equilibrium, also due to the statistical law of large numbers.

The MTPL insurance rates are determined, as mentioned, for business lines (automobiles, motorcycles, trucks, etc.) and fixed in relation to the claim costs that are assumed to be paid for business lines during the tariff's validity period. The forecast is mainly based on payments made in previous years for the same business line, on qualitative assessments, and on expert opinions identifying the outliers and the most representative years. Prior to the introduction of the direct reimbursement system, the tariff was calibrated based on claim amounts paid by the insurance company for the claims caused for each business line. Following the introduction of the direct reimbursement system, the tariff is calibrated based on the three points mentioned above and depends on both the claims policyholders incur as victims and those they are responsible for.

\subsection{Current direct reimbursement with multiple forfeits (CDRMF)}

To overcome the discrepancy that causes, as we have shown, solidarity between business lines, we hypothesize the possibility of having separate forfeits for different types of claims according to the business line of the vehicle responsible for the accident and the business line of the vehicle victim of the accident.

In this case, the forfeit would be calculated with the following formula:

$$
f_{m}^{l}=\frac{\sum_{i=1}^{n} \sum_{j=1 ; j \neq i}^{n} k(i, j)^{l, m} * c(i, j)^{l, m}}{\sum_{i=1}^{n} \sum_{j=1 ; j \neq i}^{n} k(i, j)^{l, m}} \text { with } l=1,2, \ldots, q ; m=1,2, \ldots, q .
$$

The pure premiums, in this case, would be calculated with the following formula:

$$
\begin{aligned}
* \pi_{j}^{m}= & \frac{\left[\sum_{p=1}^{q} \sum_{i=1 ; i \neq j}^{n} k(i, j)^{p, m} * c(i, j)^{p, m}+\sum_{p=1}^{q} f_{m}^{p} * \sum_{i=1 ; i \neq j}^{n} k(j, i)^{p, m}-\sum_{p=1}^{q} f_{m}^{p} * \sum_{i=1 ; i \neq j}^{n} k(i, j)^{p, m}\right]}{a_{j}^{m}} \\
& \text { with } j=1,2, \ldots, n ; m=1,2, \ldots, q
\end{aligned}
$$


and the pure market premiums will be for each $q$ business line with direct reimbursement and multiple forfeits:

$$
\begin{aligned}
\pi(\mathrm{CDRMF})_{M}^{m} & =\frac{\sum_{l=1}^{q} f_{m}^{l} * \sum_{i=1}^{n} \sum_{j=1 ; j \neq i}^{n} k(i, j)^{l, m}}{\sum_{j=1}^{n} a_{j}^{m}} \\
& =\frac{\sum_{l=1}^{q} \sum_{i=1}^{n} \sum_{j=1 ; j \neq i}^{n} k(i, j)^{l, m} * c(i, j)^{l, m}}{\sum_{j=1}^{n} a_{j}^{m}} \\
& =\pi(\mathrm{CDR})_{M}^{m} \quad \text { with } m=1,2, \ldots, q
\end{aligned}
$$

We indicate with CDRMF the current direct reimbursement with multiple forfeits.

As seen, the introduction of multiple forfeits for each type of claim (in relation to the type of vehicles involved), while modifying the premiums of single insurance companies to the market level, does not change the expected market premium with respect to the direct reimbursement system with multiple forfeits only for different business lines (current system in Italy). Clearly, when the claims occur between vehicles of the same business line, they are on average "similar" as regards the costs related to those responsible for the claim and damage. Thus, the direct reimbursement stem leads to a substantial equilibrium in costs and tariffs applied. The insurance companies charge tariffs that correspond on average to the severity of the business line that should be similar to the severity of the industrial sector for all the insurance companies.

When the claims occur between vehicles of different business lines (e.g. between a car and a motorcycle), it is apparent that, from the cost point of view, the compensations to be paid are not "similar". The motorcycle and its driver in such accidents, on average, suffer greater damage from cars and their drivers. This is irrespective of whether they are responsible for the accident or are the victim. The same consideration applies to accidents between a car and a truck, and above all, between a motorcycle and a truck. This last consideration leads to affirm that the damage suffered by a motorcycle for a claim caused by a car is, on average, greater than the damage suffered by a car for a claim caused by a motorcycle.

\subsection{Current direct reimbursement system with different forfeits (CDRDF)}

While the difference between the payments and income referred to above for motorcycles is in most cases negative and charged to the cost of claims of motorcycles, reflected in the pricing of motorcycle insurance, the difference is positive for cars. Thus, insurance companies charge tariffs that do not correspond, on average, to the accident caused by the vehicles of the business line. In fact, points $b$ and $c$ above refer to damages and not to those responsible for the claims.

To address this dysfunction, we envisage a direct reimbursement system with the forfeit mechanism only in case two vehicles belonging to the same business line are involved, while envisaging a "case by case" mechanism if the vehicles involved belong to different business lines. In the latter case, we also envisage some mechanisms to create incentives to avoid the increase in the overall system cost. 
In this case, the forfeit will be calculated with the following formula:

$$
f_{m}^{*}=\frac{\sum_{i=1}^{n} \sum_{j=1 ; j \neq i}^{n} k(i, j)^{m, m} * c(i, j)^{m, m}}{\sum_{i=1}^{n} \sum_{j=1 ; j \neq i}^{n} k(i, j)^{m, m}} \text { with } m=1,2, \ldots, q
$$

The pure premiums in this case would be calculated with the following formula where CDRDF means current direct reimbursement system with different forfeits:

$$
\begin{aligned}
* * \pi_{j}^{m}= & \frac{\left\{\sum_{i=1 ; i \neq j}^{n} k(i, j)^{m, m} * c(i, j)^{m, m}+f_{m}^{*} *\left[\sum_{i=1 ; i \neq j}^{n} k(j, i)^{m, m}-\sum_{i=1 ; i \neq j}^{n} k(i, j)^{m, m}\right]+\sum_{p=1 ; p \neq m}^{q} \sum_{i=1 ; i \neq j}^{n} k(j, i)^{m, p} * c(j, i)^{m, p}\right\}}{a_{j}^{m}} \\
& \text { with } j=1,2, \ldots, n \text { and } m=1,2, \ldots, q
\end{aligned}
$$

and market pure premiums will be for each $q$ business line with the current direct reimbursement system with different forfeits:

$$
\begin{aligned}
\pi(\mathrm{CDRDF})_{M}^{m} & =\frac{\left[\sum_{i=1}^{n} \sum_{j=1 ; j \neq i}^{n} k(i, j)^{m, m} * f_{m}^{*}+\sum_{l=1 ; l \neq m}^{q} \sum_{i=1}^{n} \sum_{j=1 ; j \neq i}^{n} k(i, j)^{m, l} * c(i, j)^{m, l}\right]}{\sum_{j=1}^{n} a_{j}^{m}} \\
& =\frac{\sum_{i=1}^{n} \sum_{j=1 ; j \neq i}^{n} \sum_{l=1}^{q} k(i, j)^{m, l} * c(i, j)^{m, l}}{\sum_{i=1}^{n} a_{i}^{l}}=\pi_{M}^{m} \quad \text { with } m=1,2, \ldots, q
\end{aligned}
$$

As this methodology shows, at the market level, we obtain the expected premium equal to what it would have been without the introduction of the direct reimbursement system. Clearly, the difference between insurance companies remains as a result of the particular claim rate of each insurance company and their ability to reimburse with lower costs than others. Obviously incentive mechanisms are necessary to motivate insurance companies to pay claims efficiently so as not to increase the premiums charged.

In this way, the general direct reimbursement mechanism (those damaged paid by their own insurance company) would remain unchanged as well as all the advantages that derive from it, e.g. speed of compensation. The only thing that would changeas described above-is the mechanism that regulates reimbursement between insurance companies (the insurance company making the compensation is assured a certain business line (e.g. paying compensation of 100 euro) and receives from the business line of the responsible party's insurance company a reimbursement in the same amount (100 euro) and not a forfeit that could be higher or lower).

\subsection{The new version of current direct reimbursement}

There is another way to overcome the bias effect described above than eliminating the forfeit mechanism of direct reimbursement when vehicles from different business lines are involved: uploading the costs incurred for the claims paid to its insured (and forfeit received) on the business line of the insured who caused the claim and not on the business line of the victim of the claim. In this way, the overall cost of the 
business line would exclusively depend on the third party liability cost of the same business line. Of course, to implement this method, insurance companies should provide information on the business line of the insured that caused the accident and that of the other insurance company.

In this case, the forfeit is calculated with the following formula:

$$
f_{l}^{*}=\frac{\sum_{m=1}^{q} \sum_{i=1}^{n} \sum_{j=1 ; j \neq i}^{n} k(i, j)^{l, m} * c(i, j)^{l, m}}{\sum_{m=1}^{q} \sum_{i=1}^{n} \sum_{j=1 ; j \neq i}^{n} k(i, j)^{l, m}} \quad \text { with } l=1,2, \ldots, q
$$

The pure premiums, in this case, would be calculated with the following formula:

$$
\begin{aligned}
\pi(\text { CDRnew })_{j}^{l}= & \frac{\left\{\sum_{m=1}^{q} \sum_{i=1 ; i \neq j}^{n} k(i, j)^{l, m} * c(i, j)^{l, m}-f_{l}^{*} *\left[\sum_{m=1}^{q} \sum_{i=1 ; i \neq j}^{n} k(j, i)^{l, m}-\sum_{m=1}^{q} \sum_{i=1 ; i \neq j}^{n} k(i, j)^{l, m}\right]\right\}}{a_{j}^{l}} \\
& \text { with } j=1,2, \ldots, n \text { and } l=1,2, \ldots, q
\end{aligned}
$$

and the pure market premiums will be for each $q$ business line:

$$
\begin{aligned}
\pi(\text { CDRnew })_{M}^{l} & =\frac{\left[\sum_{m=1}^{q} \sum_{i=1}^{n} \sum_{j=1 ; j \neq i}^{n} k(i, j)^{l, m}\right] * f_{l}^{*}}{\sum_{j=1}^{n} a_{j}^{l}} \\
& =\frac{\sum_{m=1}^{q} \sum_{i=1}^{n} \sum_{j=1 ; j \neq i}^{n} k(i, j)^{l, m} * c(i, j)^{l, m}}{\sum_{j=1}^{n} a_{j}^{l}}=\pi_{M}^{m}
\end{aligned}
$$

with $l=1,2, \ldots, q$

As is evident with this change, at the market level, there would be no distortions compared to the situation without direct reimbursement. Clearly, there would be greater differences in premiums for the single insurance companies as their average costs differ from those of the market, implying a sort of transfer of responsibility from the policyholder's insurance company to other insurance companies.

Therefore, this mechanism would lead to solidarity between insurance companies in terms of average cost of claims paid, but at the same time, would have the advantage of encouraging (almost constraining) insurance companies to align with the average market costs through an audit of costs paid for reimbursement to their insured.

\section{Empirical results and model comparison}

This section presents the results obtained by applying the various models presented in the previous section using a stochastic approach. The numerical analysis is based on the observation that the direct reimbursement mechanism always has an effect on price levels. For example, in Italy, the introduction of the direct reimbursement system has caused an increase in costs for the insured of some business lines (such 
as the $\mathrm{V}$ business line: motor vehicles): as demonstrated above, this increase is exclusively linked to the direct reimbursement operation mode described above and not to the greater risk of drivers of these vehicles. This consideration would lead to thinking that the introduction of direct reimbursement has caused a distorting effect within the MTPL business line, introducing some pricing logic for property insurance and fewer related to MTPL insurance.

We analysed the Italian data provided by CONSAP and verified empirically. In Italy in the period 2011-2014, to manage the insurance of motor vehicles, insurance companies spent about 100 million euro more compared to the costs they would have paid in the absence of the direct reimbursement system, that is, following the traditional rules. These higher costs were, of course, reflected in the premiums of the insured. Full details of this analysis can be found in Fersini et al. (2017).

Starting from these considerations, we analysed the impact of direct compensation in various possible models illustrated through a simulation and an original application of this model to automobile data from three Italian insurance companies. We carried out a numerical analysis using these data to measure the impact of the various settlement models on the level of premiums and investigate the possible distortion effect between the different sectors.

\subsection{Data description}

To simplify the operational reality and allow a univocal reading of the results, we chose to apply the models to a market in which only three companies operate. We consider the following related to the real data:

- different sizes of the three insurance companies: small, medium, and large;

- four tariff sectors: automobile and taxi (1), bus (2), truck (3), and motorcycles (4).

For each company $i$ and for each tariff sector $l$, exposure has been assumed as illustrated in Table 1.

In addition, for each company and for each tariff sector, the claim frequency and the expected cost of a claim with fault are calculated based on realistic data, and the values are shown in Tables 2 and 3, respectively.

Table 1 Number of vehicles $a_{i}^{l}$

\begin{tabular}{|c|c|c|c|c|}
\hline \multirow[t]{2}{*}{ Company } & \multicolumn{4}{|l|}{ Sector } \\
\hline & 1 & 2 & 3 & 4 \\
\hline Large & $6,600,000$ & 2000 & 750,000 & 400,000 \\
\hline Medium & $1,000,000$ & 500 & 100,000 & 100,000 \\
\hline Small & 150,000 & 150 & 5000 & 25,000 \\
\hline
\end{tabular}


Table 2 Frequency $p_{i}^{l}=\frac{s_{i}^{l}}{a_{i}^{l}}$

\begin{tabular}{|c|c|c|c|c|}
\hline \multirow[t]{2}{*}{ Company } & \multicolumn{4}{|c|}{ Sector } \\
\hline & $1(\%)$ & $2(\%)$ & $3(\%)$ & $4(\%)$ \\
\hline Large & 4.32 & 25.00 & 7.37 & 2.03 \\
\hline Medium & 4.74 & 30.60 & 8.33 & 2.27 \\
\hline Small & 6.03 & 32.00 & 9.81 & 1.88 \\
\hline
\end{tabular}

Table 3 Average cost of claims $c_{i}^{l}$

\begin{tabular}{|c|c|c|c|c|}
\hline \multirow[t]{2}{*}{ Company } & \multicolumn{4}{|l|}{ Sector } \\
\hline & 1 & 2 & 3 & 4 \\
\hline Large & 4099.07 & 5924.05 & 5523.61 & 4177.27 \\
\hline Medium & 4256.03 & 6457.22 & 5572.40 & 4177.02 \\
\hline Small & 3684.57 & 6627.53 & 2789.40 & 4907.46 \\
\hline
\end{tabular}

\subsection{Data modelling}

With $p_{i}^{l}$, we indicate the frequency of claims caused by the owners/drivers of the vehicles in sector $l$ insured by company $i$.

Therefore, in the reference period, the expected number of claims caused by owners/drivers of vehicles in sector $l$ insured by company $i$ is given by:

$$
s_{i}^{l}=a_{i}^{l} * p_{i}^{l} \quad \text { with } i=1,2, \ldots, n ; l=1,2, \ldots, q
$$

With $\alpha s t(i, j)^{l}$, we indicate the percentage distribution of the expected claims of the owners/drivers of the vehicles in sector $l$ insured by company $i$ that are instead, with the direct reimbursement system, managed by the various companies $j$.

For this hypothesis and recognizing that for $i=j \alpha s t(i, j)^{l}=0 \forall l$, then:

$$
\sum_{j=1}^{n} \alpha s t(i, j)^{l}=1 \quad \text { with } i=1,2, \ldots, n ; l=1,2, \ldots, q
$$

For each $j=1,2, \ldots, n$, we denote with $\alpha s(i, j)^{l, m}$ the percentage of expected claims of the owners/drivers of the vehicles in sector $l$ insured by company $i$ that are instead, with the direct reimbursement system, managed by the various companies $j$ related to the damaged sector $m$.

It is evident that:

$$
\sum_{m=1}^{q} \alpha s(i, j)^{l, m}=\alpha s t(i, j)^{l} \quad \forall i, l, j \text { with } i=1,2, \ldots, n ; l=1,2, \ldots, q \text { and } j=1,2, \ldots, n
$$

If for each $i=1,2, \ldots, n$ and for every $l=1,2, \ldots, q$, we multiply the expected number of claims $s_{i}^{l}$ by $\alpha s(i, j)^{l, m}$, we obtain the expected number of claims caused by the 
owners/drivers of vehicles in sector $l$ insured by company $i$ of the owners/drivers of insured vehicles of sector $m$ insured by company $j$ :

$$
\begin{aligned}
& k(i, j)^{l, m}=s_{i}^{l} * \alpha s(i, j)^{l, m} \quad \text { with } i=1,2, \ldots, n ; \\
& l=1,2, \ldots, q ; j=1,2, \ldots, n \text { and } m=1,2, \ldots, q
\end{aligned}
$$

With $\mathrm{ctm}_{i}^{l}$, we indicate the average total cost of claims caused by the owners/drivers of the vehicles in sector $l$ insured by company $i$. We define this concept as average total cost.

As is usual, we note with $c_{i}^{l}$ the average cost of each claim, so the total cost $C_{i}^{l}$ for each company $i=1,2, \ldots$, and for each sector of those responsible for the claim $l=1,2, \ldots, q$ is given by:

$$
C_{i}^{l}=s_{i}^{l} * c_{i}^{l}
$$

With $\beta c t(i, j)^{l}$, we indicate the percentage distribution of the expected total cost of claims caused by the owners/drivers of the vehicles in sector $l$ insured by company $i$ that are instead, with the direct reimbursement system, managed by various companies $j$ (it is assumed, as in reality, that every company handles claims with costs that may differ between the various companies).

For this hypothesis, and recognizing that for $i=j \beta c t(i, j)^{l}=0 \forall l$, then:

$$
\sum_{j=1}^{n} \beta c t(i, j)^{l}=1 \quad \text { with } i=1,2, \ldots, n \text { and } l=1,2, \ldots, q
$$

For each $j=1,2, \ldots, n$, we denote with $\beta c(i, j)^{l, m}$ the percentage of the expected total cost of claims of the owners/drivers of vehicles in sector $l$ insured by company $i$ that are instead, with the direct reimbursement system, managed by the various companies $j$ related to the damaged sector $m$.

It is evident that:

$$
\sum_{m=1}^{q} \beta c(i, j)^{l, m}=\beta c t(i, j)^{l} \quad \forall i, l, j \text { with } i=1,2, \ldots, n ; l=1,2, \ldots, q \text { and } j=1,2, \ldots, n
$$

If for every $i=1,2, \ldots, n$ and for every $l=1,2, \ldots, n$ we multiply the total $\cos C_{i}^{l}$ by $\beta c(i, j)^{l, m}$, we obtain the expected total cost of claims caused by owners/drivers of vehicles in sector $l$ insured by company $i$ to the owners/drivers of insured vehicles in sector $m$ insured by company $j$ :

$$
\begin{gathered}
c t(i, j)^{l, m}=C_{i}^{l} * \beta c(i, j)^{l, m} \quad \text { with } i=1,2, \ldots, n ; \\
l=1,2, \ldots, q ; j=1,2, \ldots, n \text { and } m=1,2, \ldots, q
\end{gathered}
$$

At this point, we can calculate the weighted total cost of claims caused by owners/ drivers of vehicles in sector $l$ insured by company $i$ to the owners/drivers of vehicles in sector $m$ insured by company $j$ : 


$$
\begin{gathered}
\operatorname{ctp}(i, j)^{l, m}=\operatorname{ct}(i, j)^{l, m} * k(i, j)^{l, m} \quad \text { with } i=1,2, \ldots, n ; l=1,2, \ldots, q ; j=1,2, \ldots, n \\
\text { and } m=1,2, \ldots, q
\end{gathered}
$$

from which the average total cost is:

$$
\operatorname{ctm}_{i}^{l}=\frac{\sum_{m=1}^{q} \sum_{j=1}^{n} \operatorname{ctp}(i, j)^{l, m}}{s_{i}^{l}} \quad \text { with } i=1,2, \ldots, n ; l=1,2, \ldots, q
$$

We observe that if estimating the average total cost, given the percentage of the total expected cost of claims of owners/drivers of vehicles of sector $l$ insured by company $i$ that are instead, with the direct indemnity mechanism, managed by the various companies $j$ related to the damaged sector $m, \beta c(i, j)^{l, m}$, we can easily obtain $c(i, j)^{l, m}$, i.e. the expected cost of claims caused by owners/drivers of vehicles in sector $l$ insured by company $i$ of the owners/drivers of vehicles in sector $m$ insured by company $j$. With $i=1,2, \ldots, n ; j=1,2, \ldots, n$; and $i \neq j$ and $l=1,2, \ldots, q ; m=1,2, \ldots, q$.

In fact, with the subsequent formula we obtain the weighted total cost of claims caused by owners/drivers of vehicles insured by company $i$ :

$$
\text { Ctpon }_{i}^{l}=\operatorname{ctm}_{i}^{l} * s_{i}^{l} \quad \text { with } i=1,2, \ldots, n ; l=1,2, \ldots, q
$$

If we divide $\forall i, l$, this total weighted cost in direct proportion to the percentages $\alpha s(i, j)^{l, m}$ and $\beta c(i, j)^{l, m}$ obtained is the weighted average cost $\operatorname{ctp}(i, j)^{l, m}$ from which subsequently $c t(i, j)^{l, m}$ and then $c(i, j)^{l, m}$.

$$
\begin{gathered}
c t p(i, j)^{l, m}=\frac{\operatorname{Ctpon}_{i}^{l} * \alpha s(i, j)^{l, m} * \beta c(i, j)^{l, m}}{\sum_{m=1}^{q} \sum_{j=1}^{n} \alpha s(i, j)^{l, m} * \beta c(i, j)^{l, m}} \\
c t(i, j)^{l, m}=\frac{c t p(i, j)^{l, m}}{k(i, j)^{l, m}}
\end{gathered}
$$

And therefore:

$$
c(i, j)^{l, m}=\frac{c t(i, j)^{l, m}}{k(i, j)^{l, m}}
$$

\subsection{Simulation scheme}

We developed the stochastic model with the use of simulation techniques that enable estimating both the distribution of the average cost of claims for each risk class and the distribution of index distortions. In particular, we assume that the average cost of claims is distributed according to a lognormal with mean equal to the average cost of claims for each class and a given coefficient of variation. 
To note is that for the purposes of the simulation, we assumed a coefficient of variation linked to the random variable cost of the single loss of 4 , in line with Italian market statistics.

The results of the stochastic model proposed derive from the elaboration of $1,000,000$ simulations.

From the $a_{i}^{l}$ and $p_{i}^{l}$, we obtained from the Poisson distribution the number of claims $s_{i}^{l}$ caused by the owners/drivers of vehicles in sector $l$ insured by company $i$.

From the number of claims from the data referring to the percentage of expected claims $\alpha s(i, j)^{l, m}$ (Table 4), we obtained the number of claims caused by the owners/ drivers of vehicles in sector $l$ insured by company $i$ to the owners/drivers of vehicles in sector $m$ insured by company $j$.

From the data referred to above and the data referring to the percentage of expected total cost of claims $\beta c(i, j)^{l, m}$ (Table 5), we obtained the average cost of claims caused by the owners/drivers of vehicles in sector $l$ insured by company $i$ to the owners/drivers of vehicles in sector $m$ insured by company $j$.

In particular, the data modelling described in Sect. 4.2 allows measuring via simulation the distortion caused by the introduction of the direct compensation mechanism by forfeit adjustment.

We use the following data:

1. The number of insured $a_{i}^{l}$ by each company $i$ for each sector $l$ of the driver/owner responsible.

2. The frequency of claims $p_{i}^{l}$ caused by owners/drivers of vehicles of sector $l$ insured by company $i$.

3. The percentage distribution of expected claims $\alpha s t(i, j)^{l}$ caused by the owners/ drivers of the vehicles in sector $l$ insured by company $i$ that are instead, with the direct reimbursement system, managed by the various companies $j$. With $i=j$ $\alpha s t(i, j)^{l}=0 \forall l$ and $\sum_{j=1}^{n} \alpha s t(i, j)^{l}=1$.

4. The percentage of expected claims $\alpha s(i, j)^{l, m}$ caused by the owners/drivers of the vehicles in sector $l$ insured by company $i$ that are instead, with the direct reimbursement system, managed by the various companies $j$ related to the damaged sector $m$. With $\sum_{m=1}^{q} \alpha s(i, j)^{l, m}=\alpha s t(i, j)^{l} \quad \forall i, l, j$ with $i=1,2, \ldots, n ; l=1,2, \ldots, q$ and $j=1,2, \ldots, n$.

5. The percentage distribution of the expected total cost $\beta c t(i, j)^{l}$ caused by the owners/drivers of the vehicles in sector $l$ insured by company $i$ that are instead, with the direct reimbursement system, managed by the various companies $j$. With $i=j$, $\beta c t(i, j)^{l}=0 \forall l$ and $\sum_{j=1}^{n} \beta c t(i, j)^{l}=1$.

6. The percentage of the expected total cost of claims $\beta c(i, j)^{l, m}$ caused by the owners/drivers of the vehicles in sector $l$ insured by company $i$ that are instead, with the direct reimbursement system, managed by the various companies $j$ is related to the damaged sector $m$. With $\sum_{m=1}^{q} \beta c(i, j)^{l, m}=\beta c t(i, j)^{l} \quad \forall i, l, j$ with $i=1,2, \ldots, n ; l=1,2, \ldots, q ; j=1,2, \ldots, n$.

7. The total average cost $c m_{i}^{l}$ of claims caused by the owners/drivers of vehicles of sector $l$ insured by company $i$. 
Table 4 Percentage of expected claims $\alpha s(i, j)^{l, m}$

\begin{tabular}{|c|c|c|c|c|c|}
\hline \multirow{2}{*}{$\begin{array}{l}\text { Insurance com- } \\
\text { pany of the driver } \\
\text { responsible for the } \\
\text { accident }\end{array}$} & \multirow{2}{*}{$\begin{array}{l}\text { Sector } \\
\text { of the } \\
\text { victim }\end{array}$} & \multirow{2}{*}{$\begin{array}{l}\text { Sector of the } \\
\text { driver/owner } \\
\text { responsible }\end{array}$} & \multicolumn{3}{|c|}{ Percentage of expected claims } \\
\hline & & & $\begin{array}{l}\text { Insurance } \\
\text { company of } \\
\text { victim: } 1\end{array}$ & $\begin{array}{l}\text { Insurance } \\
\text { company of } \\
\text { victim: } 2\end{array}$ & $\begin{array}{l}\text { Insurance com- } \\
\text { pany of victim: } 3\end{array}$ \\
\hline 1 & 1 & 1 & 0.0000 & 0.7310 & 0.1097 \\
\hline 1 & 2 & 1 & 0.0000 & 0.0036 & 0.0011 \\
\hline 1 & 3 & 1 & 0.0000 & 0.0921 & 0.0046 \\
\hline 1 & 4 & 1 & 0.0000 & 0.0463 & 0.0116 \\
\hline 1 & 1 & 2 & 0.0000 & 0.7406 & 0.1111 \\
\hline 1 & 2 & 2 & 0.0000 & 0.0002 & 0.0001 \\
\hline 1 & 3 & 2 & 0.0000 & 0.0952 & 0.0048 \\
\hline 1 & 4 & 2 & 0.0000 & 0.0384 & 0.0096 \\
\hline 1 & 1 & 3 & 0.0000 & 0.7358 & 0.1104 \\
\hline 1 & 2 & 3 & 0.0000 & 0.0042 & 0.0013 \\
\hline 1 & 3 & 3 & 0.0000 & 0.0921 & 0.0046 \\
\hline 1 & 4 & 3 & 0.0000 & 0.0413 & 0.0103 \\
\hline 1 & 1 & 4 & 0.0000 & 0.7170 & 0.1075 \\
\hline 1 & 2 & 4 & 0.0000 & 0.0038 & 0.0011 \\
\hline 1 & 3 & 4 & 0.0000 & 0.0921 & 0.0046 \\
\hline 1 & 4 & 4 & 0.0000 & 0.0590 & 0.0148 \\
\hline 2 & 1 & 1 & 0.8222 & 0.0000 & 0.0187 \\
\hline 2 & 2 & 1 & 0.0020 & 0.0000 & 0.0001 \\
\hline 2 & 3 & 1 & 0.1047 & 0.0000 & 0.0007 \\
\hline 2 & 4 & 1 & 0.0486 & 0.0000 & 0.0030 \\
\hline 2 & 1 & 2 & 0.8325 & 0.0000 & 0.0189 \\
\hline 2 & 2 & 2 & 0.0002 & 0.0000 & 0.0000 \\
\hline 2 & 3 & 2 & 0.0961 & 0.0000 & 0.0006 \\
\hline 2 & 4 & 2 & 0.0486 & 0.0000 & 0.0030 \\
\hline 2 & 1 & 3 & 0.8351 & 0.0000 & 0.0190 \\
\hline 2 & 2 & 3 & 0.0011 & 0.0000 & 0.0001 \\
\hline 2 & 3 & 3 & 0.0961 & 0.0000 & 0.0006 \\
\hline 2 & 4 & 3 & 0.0452 & 0.0000 & 0.0028 \\
\hline 2 & 1 & 4 & 0.8284 & 0.0000 & 0.0188 \\
\hline 2 & 2 & 4 & 0.0041 & 0.0000 & 0.0003 \\
\hline 2 & 3 & 4 & 0.0961 & 0.0000 & 0.0006 \\
\hline 2 & 4 & 4 & 0.0486 & 0.0000 & 0.0030 \\
\hline 3 & 1 & 1 & 0.8248 & 0.0187 & 0.0000 \\
\hline 3 & 2 & 1 & 0.0015 & 0.0001 & 0.0000 \\
\hline 3 & 3 & 1 & 0.0961 & 0.0006 & 0.0000 \\
\hline 3 & 4 & 1 & 0.0546 & 0.0034 & 0.0000 \\
\hline 3 & 1 & 2 & 0.8324 & 0.0189 & 0.0000 \\
\hline 3 & 2 & 2 & 0.0002 & 0.0000 & 0.0000 \\
\hline 3 & 3 & 2 & 0.0931 & 0.0006 & 0.0000 \\
\hline
\end{tabular}


Table 4 (continued)

\begin{tabular}{|c|c|c|c|c|c|}
\hline \multirow{2}{*}{$\begin{array}{l}\text { Insurance com- } \\
\text { pany of the driver } \\
\text { responsible for the } \\
\text { accident }\end{array}$} & \multirow{2}{*}{$\begin{array}{l}\text { Sector } \\
\text { of the } \\
\text { victim }\end{array}$} & \multirow{2}{*}{$\begin{array}{l}\text { Sector of the } \\
\text { driver/owner } \\
\text { responsible }\end{array}$} & \multicolumn{3}{|c|}{ Percentage of expected claims } \\
\hline & & & $\begin{array}{l}\text { Insurance } \\
\text { company of } \\
\text { victim: } 1\end{array}$ & $\begin{array}{l}\text { Insurance } \\
\text { company of } \\
\text { victim: } 2\end{array}$ & $\begin{array}{l}\text { Insurance com- } \\
\text { pany of victim: } 3\end{array}$ \\
\hline 3 & 4 & 2 & 0.0515 & 0.0032 & 0.0000 \\
\hline 3 & 1 & 3 & 0.8243 & 0.0187 & 0.0000 \\
\hline 3 & 2 & 3 & 0.0019 & 0.0001 & 0.0000 \\
\hline 3 & 3 & 3 & 0.0982 & 0.0007 & 0.0000 \\
\hline 3 & 4 & 3 & 0.0528 & 0.0033 & 0.0000 \\
\hline 3 & 1 & 4 & 0.8012 & 0.0182 & 0.0000 \\
\hline 3 & 2 & 4 & 0.0049 & 0.0004 & 0.0000 \\
\hline 3 & 3 & 4 & 0.1055 & 0.0007 & 0.0000 \\
\hline 3 & 4 & 4 & 0.0650 & 0.0041 & 0.0000 \\
\hline
\end{tabular}

8. The standard deviation of the average total cost $s q m_{i}^{l}$ of claims caused by the owners/drivers vehicles of sector $l$ insured by company $i$.

Through these data, we implemented the simulation scheme by performing the following steps, replicated 1,000,000 times:

- With the data referred to in points 1 and 2, we obtained from the Poisson distribution the number of claims $s_{i}^{l}$ caused by the owners/drivers of the vehicles in sector $l$ insured by company $i$.

- From the number of claims $s_{i}^{l}$, from the data referred to in points 3 and 4 , we obtained the number of claims caused by the owners/drivers of the vehicles in sector $l$ insured by company $i$ to the owners/drivers of the vehicles in sector $m$ insured by company $j k(i, j)^{l, m}$.

- From the data referred to in points 3-8 and the data referred to in point 2, we obtained the average cost of claims caused by the owners/drivers of the vehicles in sector $l$ insured by company $i$ to the owners/drivers of the vehicles in sector $m$ insured by company $j$. In particular, we used a Log Normal Distribution to obtain from points 7 and 8 the average total cost.

- From $k(i, j)^{l, m}$ and $c(i, j)^{l, m}$, we obtained the mean values and distributions of the quantities referred to in this paper.

\subsection{Results and model comparison}

The purpose of the numerical application below is to calculate the pure premium for each sector based on the different direct compensation models described in the previous section and to evaluate the distortion in terms of different levels of pure premiums with respect to traditional reimbursement without direct compensation. 
Table 5 Percentage of the expected total cost of claims $\beta c(i, j)^{l, m}$

\begin{tabular}{|c|c|c|c|c|c|}
\hline \multirow{2}{*}{$\begin{array}{l}\text { Insurance com- } \\
\text { pany of the driver } \\
\text { responsible for the } \\
\text { accident }\end{array}$} & \multirow{2}{*}{$\begin{array}{l}\text { Sector } \\
\text { of the } \\
\text { victim }\end{array}$} & \multirow{2}{*}{$\begin{array}{l}\text { Sector of the } \\
\text { driver/owner } \\
\text { responsible }\end{array}$} & \multicolumn{3}{|c|}{ Percentage of expected total cost } \\
\hline & & & $\begin{array}{l}\text { Insurance } \\
\text { company of } \\
\text { victim: } 1\end{array}$ & $\begin{array}{l}\text { Insurance } \\
\text { company of } \\
\text { victim: } 2\end{array}$ & $\begin{array}{l}\text { Insurance com- } \\
\text { pany of victim: } 3\end{array}$ \\
\hline 1 & 1 & 1 & 0.0000 & 0.6919 & 0.1038 \\
\hline 1 & 2 & 1 & 0.0000 & 0.0030 & 0.0009 \\
\hline 1 & 3 & 1 & 0.0000 & 0.0848 & 0.0042 \\
\hline 1 & 4 & 1 & 0.0000 & 0.0891 & 0.0223 \\
\hline 1 & 1 & 2 & 0.0000 & 0.7076 & 0.1061 \\
\hline 1 & 2 & 2 & 0.0000 & 0.0002 & 0.0001 \\
\hline 1 & 3 & 2 & 0.0000 & 0.0885 & 0.0044 \\
\hline 1 & 4 & 2 & 0.0000 & 0.0746 & 0.0186 \\
\hline 1 & 1 & 3 & 0.0000 & 0.7008 & 0.1051 \\
\hline 1 & 2 & 3 & 0.0000 & 0.0035 & 0.0011 \\
\hline 1 & 3 & 3 & 0.0000 & 0.0853 & 0.0043 \\
\hline 1 & 4 & 3 & 0.0000 & 0.0799 & 0.0200 \\
\hline 1 & 1 & 4 & 0.0000 & 0.6683 & 0.1002 \\
\hline 1 & 2 & 4 & 0.0000 & 0.0031 & 0.0009 \\
\hline 1 & 3 & 4 & 0.0000 & 0.0835 & 0.0042 \\
\hline 1 & 4 & 4 & 0.0000 & 0.1118 & 0.0280 \\
\hline 2 & 1 & 1 & 0.7829 & 0.0000 & 0.0178 \\
\hline 2 & 2 & 1 & 0.0017 & 0.0000 & 0.0001 \\
\hline 2 & 3 & 1 & 0.0970 & 0.0000 & 0.0006 \\
\hline 2 & 4 & 1 & 0.0940 & 0.0000 & 0.0059 \\
\hline 2 & 1 & 2 & 0.7924 & 0.0000 & 0.0180 \\
\hline 2 & 2 & 2 & 0.0002 & 0.0000 & 0.0000 \\
\hline 2 & 3 & 2 & 0.0889 & 0.0000 & 0.0006 \\
\hline 2 & 4 & 2 & 0.0939 & 0.0000 & 0.0059 \\
\hline 2 & 1 & 3 & 0.7978 & 0.0000 & 0.0181 \\
\hline 2 & 2 & 3 & 0.0009 & 0.0000 & 0.0001 \\
\hline 2 & 3 & 3 & 0.0893 & 0.0000 & 0.0006 \\
\hline 2 & 4 & 3 & 0.0877 & 0.0000 & 0.0055 \\
\hline 2 & 1 & 4 & 0.7889 & 0.0000 & 0.0179 \\
\hline 2 & 2 & 4 & 0.0035 & 0.0000 & 0.0003 \\
\hline 2 & 3 & 4 & 0.0890 & 0.0000 & 0.0006 \\
\hline 2 & 4 & 4 & 0.0940 & 0.0000 & 0.0059 \\
\hline 3 & 1 & 1 & 0.7804 & 0.0177 & 0.0000 \\
\hline 3 & 2 & 1 & 0.0013 & 0.0001 & 0.0000 \\
\hline 3 & 3 & 1 & 0.0884 & 0.0006 & 0.0000 \\
\hline 3 & 4 & 1 & 0.1050 & 0.0066 & 0.0000 \\
\hline 3 & 1 & 2 & 0.7899 & 0.0180 & 0.0000 \\
\hline 3 & 2 & 2 & 0.0002 & 0.0000 & 0.0000 \\
\hline 3 & 3 & 2 & 0.0859 & 0.0006 & 0.0000 \\
\hline
\end{tabular}


Table 5 (continued)

\begin{tabular}{|c|c|c|c|c|c|}
\hline \multirow{2}{*}{$\begin{array}{l}\text { Insurance com- } \\
\text { pany of the driver } \\
\text { responsible for the } \\
\text { accident }\end{array}$} & \multirow{2}{*}{$\begin{array}{l}\text { Sector } \\
\text { of the } \\
\text { victim }\end{array}$} & \multirow{2}{*}{$\begin{array}{l}\text { Sector of the } \\
\text { driver/owner } \\
\text { responsible }\end{array}$} & \multicolumn{3}{|c|}{ Percentage of expected total cost } \\
\hline & & & $\begin{array}{l}\text { Insurance } \\
\text { company of } \\
\text { victim: } 1\end{array}$ & $\begin{array}{l}\text { Insurance } \\
\text { company of } \\
\text { victim: } 2\end{array}$ & $\begin{array}{l}\text { Insurance com- } \\
\text { pany of victim: } 3\end{array}$ \\
\hline 3 & 4 & 2 & 0.0993 & 0.0062 & 0.0000 \\
\hline 3 & 1 & 3 & 0.7815 & 0.0178 & 0.0000 \\
\hline 3 & 2 & 3 & 0.0016 & 0.0001 & 0.0000 \\
\hline 3 & 3 & 3 & 0.0905 & 0.0006 & 0.0000 \\
\hline 3 & 4 & 3 & 0.1016 & 0.0064 & 0.0000 \\
\hline 3 & 1 & 4 & 0.7504 & 0.0171 & 0.0000 \\
\hline 3 & 2 & 4 & 0.0041 & 0.0003 & 0.0000 \\
\hline 3 & 3 & 4 & 0.0961 & 0.0006 & 0.0000 \\
\hline 3 & 4 & 4 & 0.1237 & 0.0077 & 0.0000 \\
\hline
\end{tabular}

We show in Fig. 1 the empirical distributions of the pure premium level using the data of the three insurance companies in the absence of direct compensation for each sector.

Table 6 shows, as an example, the pure premiums (mean) for sector 1 and for the three insurance companies without DR and with current DR, and the main statistics of interest (standard deviation, percentiles) regarding the expected aggregate claim for the single risk.
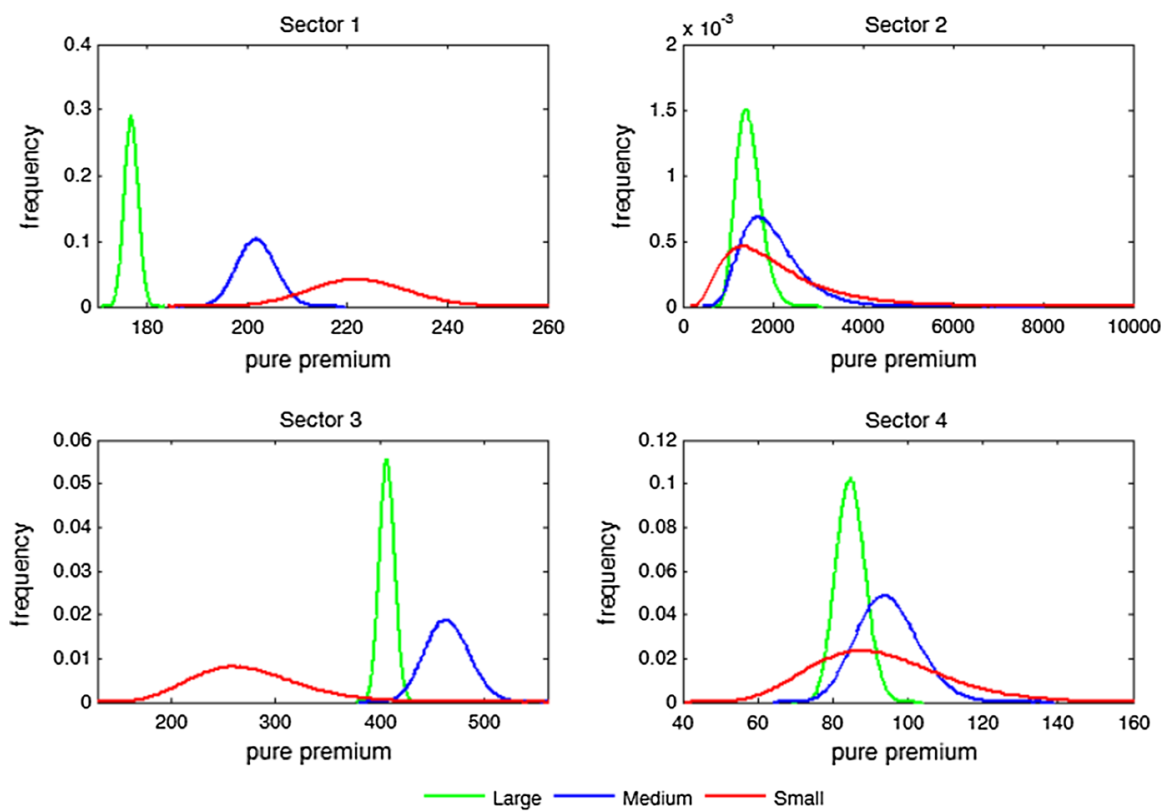

Fig. 1 Pure premium distribution 
Table 6 Pure premium and statistics-sector 1

\begin{tabular}{|c|c|c|c|c|c|c|}
\hline & \multicolumn{3}{|c|}{ Without DR } & \multicolumn{3}{|l|}{ CDR } \\
\hline & Large & Medium & Small & Large & Medium & Small \\
\hline \multicolumn{7}{|l|}{ Sector 1} \\
\hline $\begin{array}{l}\text { Pure pre- } \\
\text { mium- } \\
\text { mean }\end{array}$ & 176.90 & 201.81 & 222.33 & 182.79 & 200.08 & 231.81 \\
\hline Min & 170.89 & 185.42 & 183.90 & 176.82 & 182.18 & 212.94 \\
\hline $\operatorname{Max}$ & 183.52 & 219.63 & 269.19 & 189.18 & 216.00 & 251.17 \\
\hline Median & 176.89 & 201.77 & 222.12 & 182.79 & 200.09 & 231.80 \\
\hline STD & $137 \%$ & $382 \%$ & $962 \%$ & $131 \%$ & $357 \%$ & $394 \%$ \\
\hline \multicolumn{7}{|l|}{ Percentile } \\
\hline $10 \%$ & 175.14 & 196.90 & 210.07 & 181.11 & 195.51 & 226.76 \\
\hline $20 \%$ & 175.74 & 198.55 & 214.10 & 181.69 & 197.08 & 228.49 \\
\hline $30 \%$ & 176.17 & 199.75 & 217.07 & 182.10 & 198.21 & 229.74 \\
\hline $40 \%$ & 176.55 & 200.79 & 219.67 & 182.46 & 199.18 & 230.81 \\
\hline $50 \%$ & 176.89 & 201.77 & 222.12 & 182.79 & 200.09 & 231.80 \\
\hline $60 \%$ & 177.25 & 202.75 & 224.60 & 183.12 & 200.99 & 232.80 \\
\hline $70 \%$ & 177.62 & 203.81 & 227.27 & 183.47 & 201.96 & 233.87 \\
\hline $80 \%$ & 178.06 & 205.05 & 230.45 & 183.89 & 203.09 & 235.13 \\
\hline $90 \%$ & 178.67 & 206.76 & 234.87 & 184.47 & 204.67 & 236.87 \\
\hline $100 \%$ & 183.52 & 219.63 & 269.19 & 189.18 & 216.00 & 251.17 \\
\hline
\end{tabular}

If we compare the differences of the mean for sector 1 , which is the most important in MTPL insurance, these differences are small for all insurance companies analysed, meaning that the distortion introduced by the DR scheme has no deep effects on sector 1, which represents more than $90 \%$ of risk. On average, the introduction of the CDR system has led to a slight increase in the pure premium for sector 1 .

Table 7 shows, for the medium insurance company, the pure premiums for all sectors without DR and with DR (CDR) and all the most important statistics regarding the expected aggregate claim for the single risk.

As we can see, for the medium insurance company:

- for sector 1, the distortions are small, a pure premium from 201.81 to 200.08, since automobiles and taxis constitute almost the total risk in the medium insurance company portfolio;

- for sector 2, buses, the distortions are considerable, a pure premium from 1976.03 to 884.64 ; this means that following the introduction of DR, the pure premium has strongly decreased;

- for sector 3, trucks, the distortions are considerable, a pure premium from 464.31 to 239.76; this means that following the introduction of $\mathrm{DR}$, the pure premium has strongly decreased; 
Table 7 Pure premium and statistics all sectors-medium insurance company

\begin{tabular}{|c|c|c|c|c|c|c|c|c|}
\hline & \multicolumn{4}{|c|}{ Without DR } & \multicolumn{4}{|l|}{ CDR } \\
\hline & Sector 1 & Sector 2 & Sector 3 & Sector 4 & Sector 1 & Sector 2 & Sector 3 & Sector 4 \\
\hline \multicolumn{9}{|l|}{ Medium } \\
\hline $\begin{array}{c}\text { Pure pre- } \\
\text { mium- } \\
\text { mean }\end{array}$ & 201.81 & 1976.03 & 464.31 & 94.71 & 200.08 & 884.64 & 239.76 & 244.73 \\
\hline Min & 185.42 & 451.95 & 379.40 & 63.94 & 182.18 & 802.49 & 217.31 & 223.37 \\
\hline $\operatorname{Max}$ & 219.63 & 8048.26 & 568.17 & 139.37 & 216.00 & 967.01 & 259.91 & 263.68 \\
\hline Median & 201.77 & 1874.31 & 463.82 & 94.36 & 200.09 & 884.63 & 239.76 & 244.73 \\
\hline STD & $382 \%$ & $65,721 \%$ & $2096 \%$ & $819 \%$ & $357 \%$ & $1653 \%$ & $449 \%$ & $422 \%$ \\
\hline \multicolumn{9}{|l|}{ Percentile } \\
\hline $10 \%$ & 196.90 & 1233.19 & 437.67 & 84.41 & 195.51 & 863.44 & 234.00 & 239.31 \\
\hline $20 \%$ & 198.55 & 1422.53 & 446.42 & 87.67 & 197.08 & 870.71 & 235.98 & 241.17 \\
\hline $30 \%$ & 199.75 & 1577.74 & 452.87 & 90.13 & 198.21 & 875.96 & 237.40 & 242.51 \\
\hline $40 \%$ & 200.79 & 1724.85 & 458.46 & 92.28 & 199.18 & 880.45 & 238.62 & 243.66 \\
\hline $50 \%$ & 201.77 & 1874.31 & 463.82 & 94.36 & 200.09 & 884.63 & 239.76 & 244.73 \\
\hline $60 \%$ & 202.75 & 2036.97 & 469.23 & 96.47 & 200.99 & 888.83 & 240.91 & 245.81 \\
\hline $70 \%$ & 203.81 & 2226.82 & 475.05 & 98.78 & 201.96 & 893.34 & 242.12 & 246.95 \\
\hline $80 \%$ & 205.05 & 2469.91 & 481.95 & 101.55 & 203.09 & 898.57 & 243.55 & 248.28 \\
\hline $90 \%$ & 206.76 & 2849.46 & 491.64 & 105.46 & 204.67 & 905.86 & 245.53 & 250.15 \\
\hline $100 \%$ & 219.63 & 8048.26 & 568.17 & 139.37 & 216.00 & 967.01 & 259.91 & 263.68 \\
\hline
\end{tabular}

- for sector 4, motorcycles, the distortions are considerable, a pure premium from 94.71 to 244.73 ; this means that following the introduction of DR, the pure premium has strongly increased.

As can be seen from the table above, the greatest distortion is recorded for sector 4 with an increase in the premium of $158 \%$. This result is due, as anticipated, to the fact that the motor vehicle sector is characterized by low costs for claims caused and high costs for the claim victims; therefore, with CDR, this sector absorbs part of the costs of the motor vehicle third party insurance of other sectors.

Table 8 shows, for the market, the pure premiums for all sectors with and without DR and the most relevant statistics regarding the expected aggregate claim for the single risk.

As we can see, from a market point of view:

- for sector 1, the distortions are small, a pure premium from 180.99 to 185.97 , since automobiles and taxis constitute almost the total risk in the market portfolio;

- for sector 2, buses, the distortions are considerable, a pure premium from 1610.66 to 2479.17 ; this means that following the introduction of DR, the pure premium has strongly increased; 
Table 8 Pure market premium and statistics all sectors-comparison with and without DR

\begin{tabular}{|c|c|c|c|c|c|c|c|c|}
\hline & \multicolumn{4}{|c|}{ Without DR } & \multicolumn{4}{|l|}{ CDR } \\
\hline & Sector 1 & Sector 2 & Sector 3 & Sector 4 & Sector 1 & Sector 2 & Sector 3 & Sector 4 \\
\hline \multicolumn{9}{|l|}{ Market } \\
\hline $\begin{array}{c}\text { Pure pre- } \\
\text { mium- } \\
\text { mean }\end{array}$ & 180.99 & 1610.66 & 413.06 & 87.14 & 185.97 & 2479.17 & 190.30 & 372.07 \\
\hline Min & 175.21 & 745.51 & 383.30 & 73.18 & 180.36 & 2398.46 & 184.58 & 361.00 \\
\hline Max & 187.02 & 3257.18 & 445.13 & 104.03 & 191.90 & 2557.82 & 196.40 & 383.76 \\
\hline Median & 180.99 & 1591.90 & 413.01 & 87.07 & 185.97 & 2479.11 & 190.29 & 372.07 \\
\hline STD & $128 \%$ & $25,046 \%$ & $674 \%$ & $345 \%$ & $120 \%$ & $1662 \%$ & $123 \%$ & $239 \%$ \\
\hline \multicolumn{9}{|l|}{ Percentile } \\
\hline $10 \%$ & 179.35 & 1304.39 & 404.42 & 82.75 & 184.43 & 2457.87 & 188.72 & 369.01 \\
\hline $20 \%$ & 179.91 & 1396.06 & 407.33 & 84.20 & 184.96 & 2465.13 & 189.26 & 370.06 \\
\hline $30 \%$ & 180.32 & 1466.35 & 409.46 & 85.27 & 185.34 & 2470.38 & 189.65 & 370.81 \\
\hline $40 \%$ & 180.66 & 1529.93 & 411.29 & 86.19 & 185.66 & 2474.87 & 189.98 & 371.46 \\
\hline $50 \%$ & 180.99 & 1591.90 & 413.01 & 87.07 & 185.97 & 2479.11 & 190.29 & 372.07 \\
\hline $60 \%$ & 181.32 & 1655.92 & 414.74 & 87.95 & 186.27 & 2483.36 & 190.60 & 372.67 \\
\hline $70 \%$ & 181.67 & 1727.33 & 416.58 & 88.91 & 186.60 & 2487.88 & 190.94 & 373.32 \\
\hline $80 \%$ & 182.07 & 1814.38 & 418.75 & 90.03 & 186.98 & 2493.19 & 191.33 & 374.09 \\
\hline $90 \%$ & 182.64 & 1941.65 & 421.76 & 91.62 & 187.51 & 2500.57 & 191.87 & 375.14 \\
\hline $100 \%$ & 187.02 & 3257.18 & 445.13 & 104.03 & 191.90 & 2557.82 & 196.40 & 383.76 \\
\hline
\end{tabular}

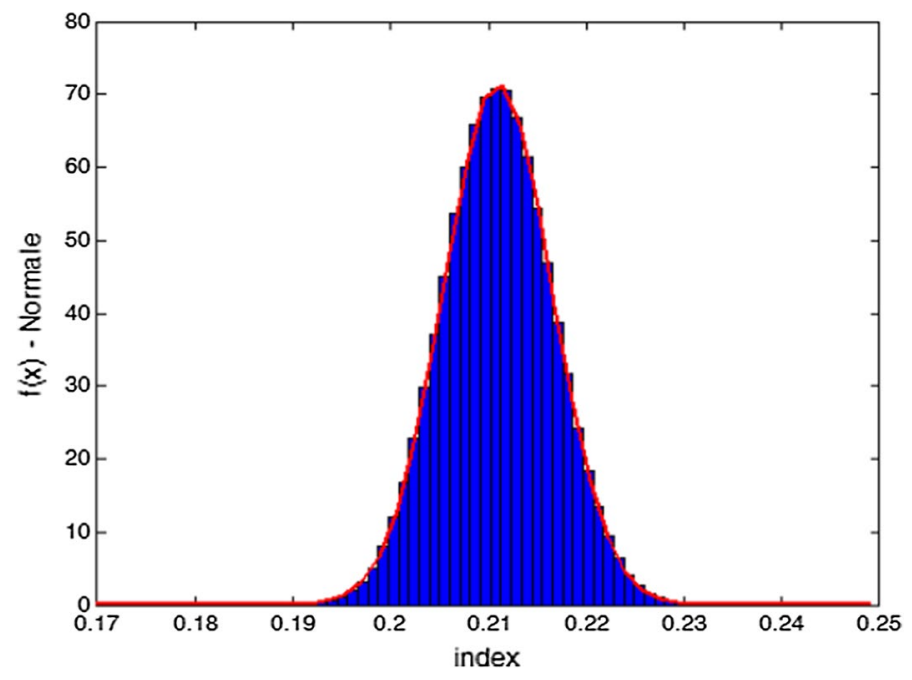

Fig. 2 Market solidarity index distribution 
Table 9 Market solidarity index

\begin{tabular}{ll}
\hline Market & \\
Pure premium-mean & $21.09 \%$ \\
Min & $18.62 \%$ \\
Max & $23.85 \%$ \\
Median & $21.09 \%$ \\
STD & $0.56 \%$ \\
Percentile & \\
$10 \%$ & $20.38 \%$ \\
$20 \%$ & $20.62 \%$ \\
$30 \%$ & $20.80 \%$ \\
$40 \%$ & $20.95 \%$ \\
$50 \%$ & $21.09 \%$ \\
$60 \%$ & $21.23 \%$ \\
$70 \%$ & $21.38 \%$ \\
$80 \%$ & $21.56 \%$ \\
$90 \%$ & $21.81 \%$ \\
$100 \%$ & $23.85 \%$ \\
\hline
\end{tabular}
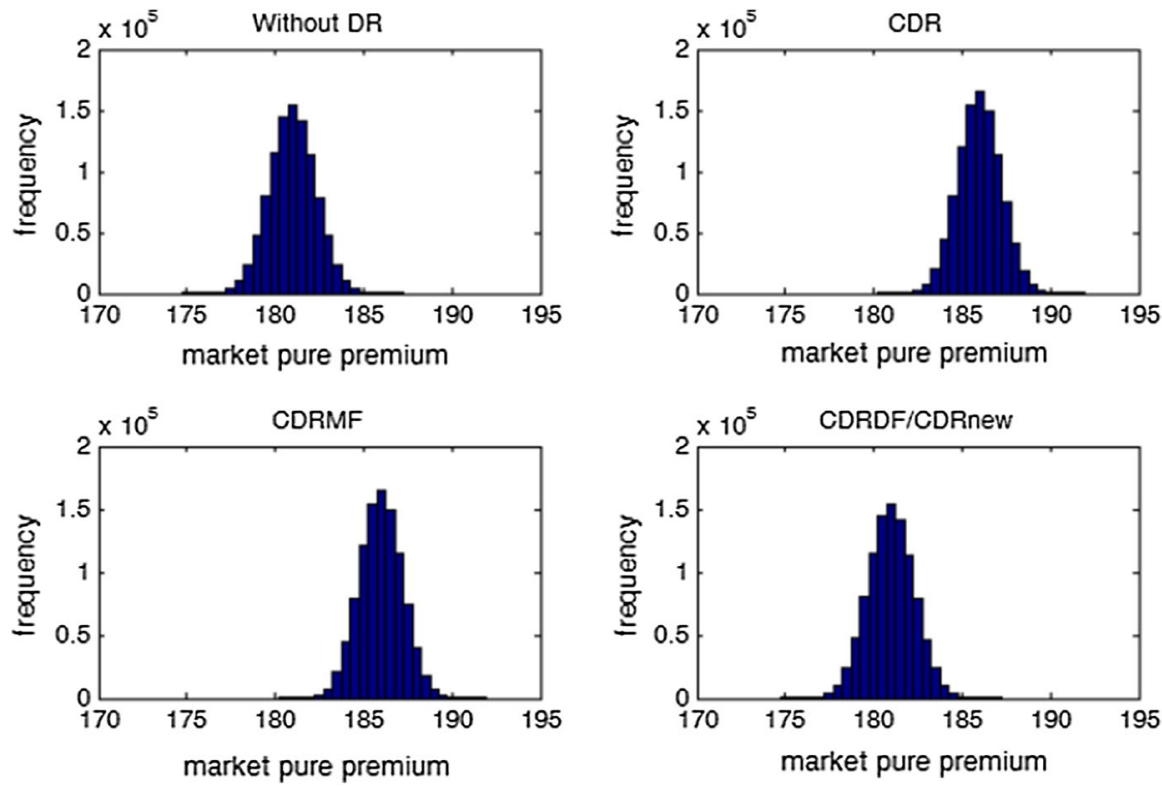

Fig. 3 Pure market premium empirical distribution —-sector 1 -all methods

- for sector 3, trucks, the distortions are considerable, a pure premium from 413.06 to 190.30; this means that following the introduction of DR, the pure premium has strongly decreased in line with the medium company; 

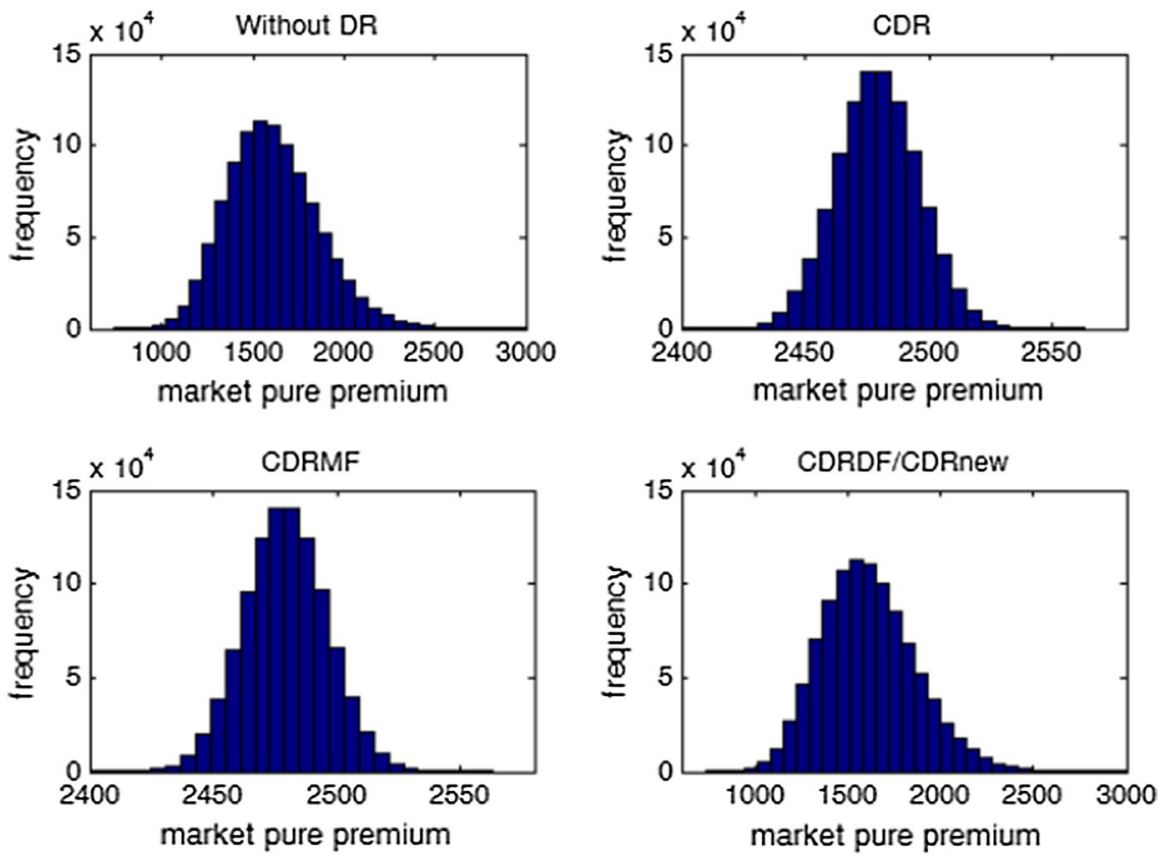

Fig. 4 Pure market premium empirical distribution-sector 2-all methods
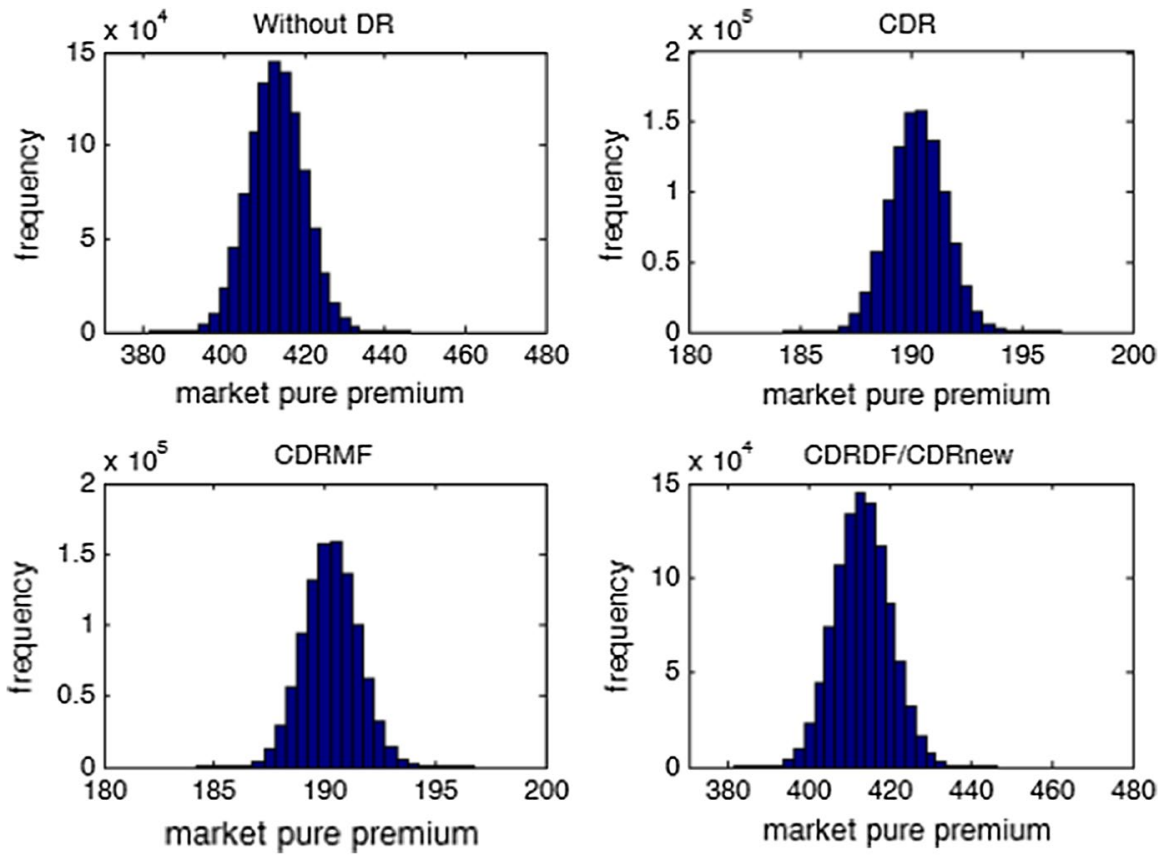

Fig. 5 Pure market premium empirical distribution—sector 3-all methods 

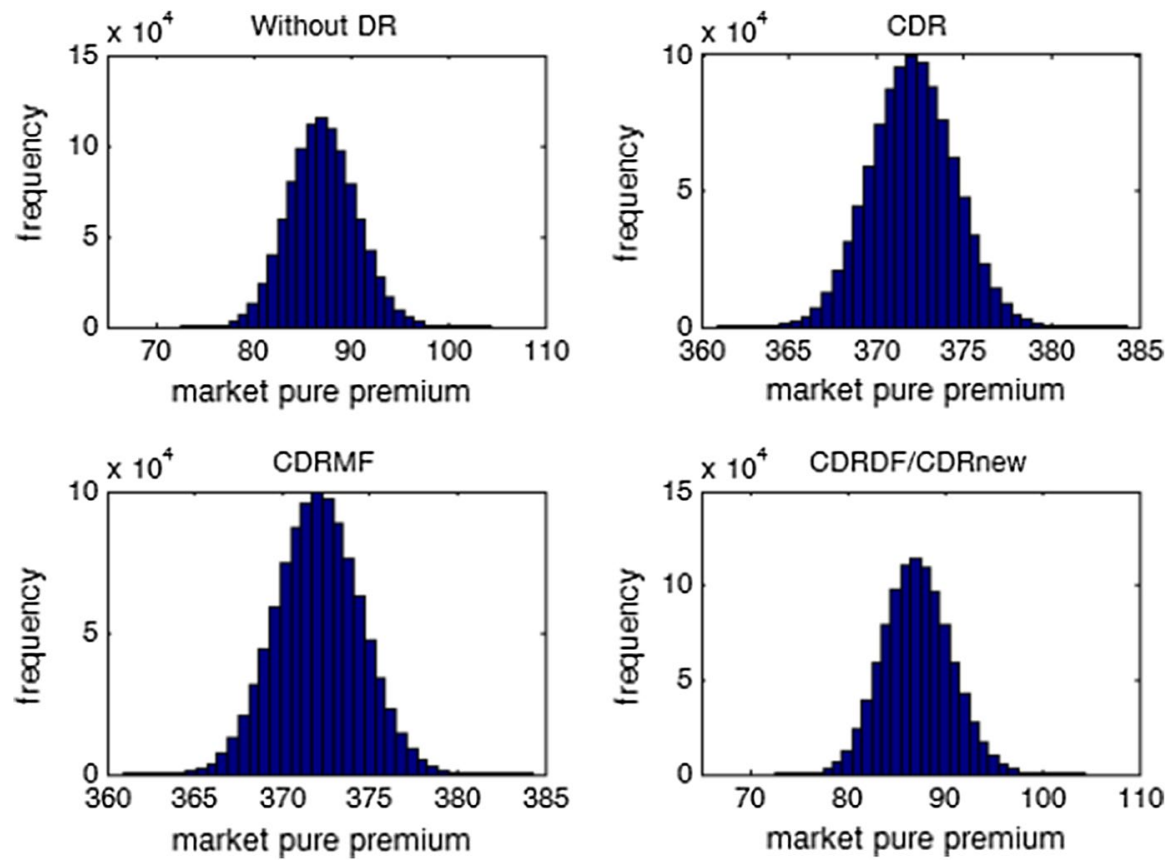

Fig. 6 Pure market premium empirical distribution—sector 4—all methods

- for sector 4, motorcycles, the distortions are considerable, a pure premium from 87.14 to 372.07 ; this means that following the introduction of DR, the pure premium has strongly increased in line with the medium company.

In line with the medium company, the market shows the strongest distortion for sector 4 , equal to $327 \%$.

Figure 2 and Table 9 show the simulated distribution and the statistics of the market solidarity index that we defined in (10).

As we can see, after the introduction of the current DR, at the market level, on average for a specific sector for every 100 euro of premium we have more or less 21.09 euro with respect to the pure premium without DR.

The following is a comparison of the results obtained for all the models analysed.

As evidenced in the preceding table, if we take the CDRDF and new DR scheme and apply formulas (16) and (19), we obtain the exact pure premium for the market that we obtained without DR (reported in Table 8). With the CDRMF scheme, we obtain the same pure market premium values as with the current DR (CDR), so at the market level, there is no benefit as the distortion remains.

For greater detail and a better understanding of the comparisons between the market premiums of the different systems, below we report the simulated distributions of the pure market premiums for the 4 sectors.

As evidenced in the preceding graphs (Figs. 3, 4, 5, 6), in the case of the CDRDF or new CDR scheme, we obtain the same pure market premium distribution as without DR. 


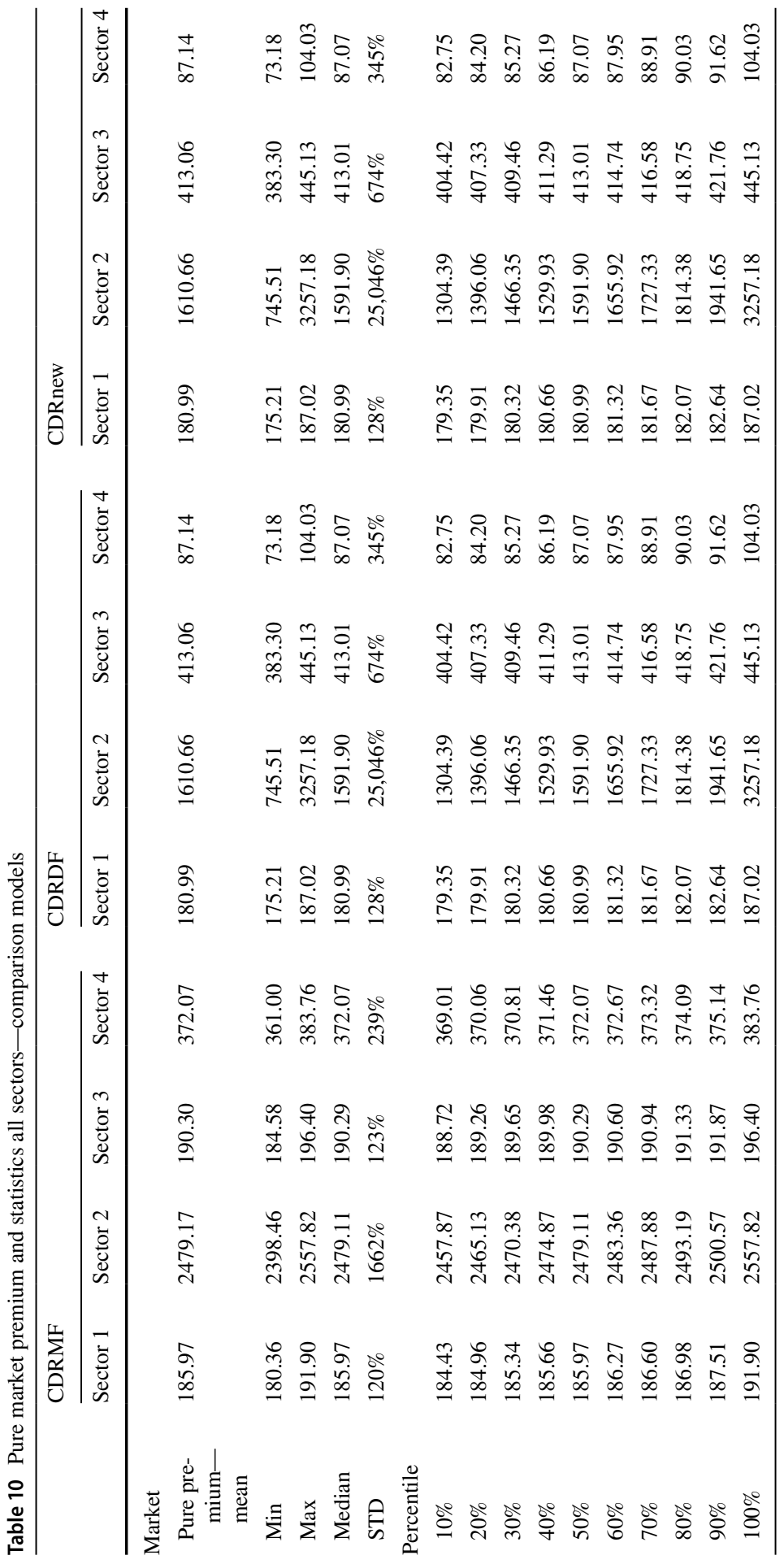


Table 11 Pure premium and statistics all sectors-new CDR method-medium company

\begin{tabular}{lllll}
\hline & Sector 1 & Sector 2 & Sector 3 & Sector 4 \\
\hline $\begin{array}{c}\text { Medium } \\
\begin{array}{c}\text { Pure pre- } \\
\text { mium- }\end{array}\end{array}$ & 187.17 & 1699.97 & 453.99 & 91.54 \\
$\quad$ mean & & & & \\
Min & 169.29 & -1616.15 & 359.96 & 52.96 \\
Max & 204.56 & 5474.32 & 551.45 & 132.51 \\
Median & 187.17 & 1674.95 & 454.07 & 91.47 \\
STD & $381 \%$ & $64,239 \%$ & $2060 \%$ & $841 \%$ \\
Percentile & & & & \\
$10 \%$ & 182.28 & 908.69 & 427.48 & 80.81 \\
$20 \%$ & 183.96 & 1168.24 & 436.60 & 84.42 \\
$30 \%$ & 185.16 & 1356.13 & 443.18 & 87.07 \\
$40 \%$ & 186.20 & 1519.56 & 448.82 & 89.34 \\
$50 \%$ & 187.17 & 1674.95 & 454.07 & 91.47 \\
$60 \%$ & 188.14 & 1834.08 & 459.31 & 93.61 \\
$70 \%$ & 189.18 & 2009.20 & 464.88 & 95.91 \\
$80 \%$ & 190.39 & 2220.78 & 471.35 & 98.61 \\
$90 \%$ & 192.07 & 2527.82 & 480.36 & 102.39 \\
$100 \%$ & 204.56 & 5474.32 & 551.45 & 132.51 \\
\hline
\end{tabular}

In Table 11, as an example, we present the results for the medium company by sector in the case of the new CRD scheme.

As shown, at the market level, the new CDR method does not entail any distortion (see the comparison of the levels of pure premium in Table 10 with the corresponding values in Table 7). At each company level (in Table 11, as an example, the medium company is reported), the distortion remains for the single sector, but is much smaller with respect to the other methods reported. In particular, for sector 4, the variation can be considered small; in fact, comparing the values in Table 11 with those in Table 7, the pure premium in the system without DR is equal to 94.71, and with CDR it increases to 244.73, and in the case of the new CDR, it results in a slight reduction equal to 91.54 . Moreover, this reduction is due to the fact that the pure premium is influenced by the claims in the portfolios of the other companies. In the specific case of the pure premium of the medium company, being largely dependent on the claims of the large company, and assuming for the latter a lower frequency and average costs, it is lower with respect to the premium that the medium company would have in the absence of DR, that is, calculated only on its own risk portfolio, thus enjoying an advantage in terms of competitiveness.

\section{Conclusion}

In a system of motor vehicle third party liability with a direct compensation mechanism, the injured party is compensated by his/her insurance company instead of the insurance company of the person causing the accident. Subsequently, the companies 
regulate their relationship through a compensation mechanism. The mechanism under examination would allow the limits of RCA insurance to be exceeded, linked to the third party of the injured party and reduced litigation, thus reproducing the advantages of the no-fault system in a third party liability regime.

In some countries, such as Italy and France, an ex-ante offsetting mechanism is envisaged between companies, which determines the average cost, hence the forfeit, taking into account the compensation actually paid by the companies of the entire market in the previous year. This system has led to some problems. In fact, the forfeit compensation method has brought some critical elements for companies, since it does not allow distinguishing between vehicles that have different characteristics in the claims, both in terms of frequency of accidents and incidence of damage to the person or their property. This problem is significant for all categories of motor vehicles, but has strikingly emerged in the case of motor vehicles, characterized by high incidence of physical damage and an average cost of the accident victim greater than the average overall cost, and above all, a large difference between the cost of the claims suffered and the cost of the accidents caused.

The authors began their statistical analysis following a principle of economic theory: the introduction of direct compensation leads to a reduction in damage settlement times, the reduction in the cost of driving should lead to an increase in driving activity, and the reduction in the cost of insurance premiums should lead to an increase in car owners (Brown 1985). Starting from this principle and analysing the statistical data provided by CONSAP, we found that in the Italian market, the introduction of the DR scheme has introduced some distortion effects specifically for trucks and motorcycles in the period 2011-2014.

In this paper, we analysed a generic market, simulating through a stochastic model the effects of the introduction of different DR and determining an average distortion equal to $21.09 \%$. Considering that automobiles represent the main sector, this distortion, which might not seem considerable, is, however, huge for trucks and motorcycles.

We propose a double solution to the problem:

(A) if applying CDRDF, considering only the accidents where vehicles of the same sector are involved, thus the distortion at market level is null;

(B) if applying the new CDR, considering all accidents, the distortion at market level and insurance company level is not considerable even if insurance companies in this case should have more information about the accident, for instance, the sector of the vehicle that caused the claims.

Moreover, the new CDR system proposed, at the single company level, on the one hand overcomes the distortion between sectors because each sector absorbs its responsibility without affecting that relating to other sectors; on the other hand, it highlights a sort of "solidarity" between companies, implying the levels of premiums are correlated with the claims of the risk portfolio of the same sector but other companies. 
It remains to be noted that direct compensation does not undermine the trilateral structure of the third party liability insurance - the damaged, the damaging, and the insurer-and therefore does not alter the third party insurance system (Carriero 2009). This is confirmed by the fact that, in the direct reimbursement system, the insurer that manages the claim acts on behalf of the debtor subrogating it.

The advantages linked to establishing a direct relationship between the insured damaged and the insurer are instead typical of first party insurance, where a contractual relationship is established between the damaged and the insurer.

In any case, there are many criticisms of those systems that have introduced a mechanism that stands as an intermediate model between first party insurance and third party insurance attempting, with poor results, to replicate the advantages of the first party in a third party system.

Are the reasons that led the European legislature to prefer a system of compulsory liability through the Strasbourg Convention (European Convention on Compulsory Insurance against Third Party Liability with respect to Motor Vehicles), signed in Strasbourg in 1959) over direct insurance (considered too expensive) still valid today?

Open Access This article is distributed under the terms of the Creative Commons Attribution 4.0 International License (http://creativecommons.org/licenses/by/4.0/), which permits unrestricted use, distribution, and reproduction in any medium, provided you give appropriate credit to the original author(s) and the source, provide a link to the Creative Commons license, and indicate if changes were made.

\section{References}

Born, P.: The influence of Tort reform on auto liability losses and premiums. J. Insur. Issues 40(1), 61-89 (2017)

Bouzouita, R., Bajtelsmit, V.L.: The impact of rate regulation on the residual market for automobile insurance. J. Insur. Regul. 16(1), 61-72 (1997)

Brown, C.: Deterrence in Tort and no-fault: the New Zealand experience. Calif. Law Rev. 3, 976-1002 (1985)

Carriero, G.: L'indennizzo diretto. Assicurazioni 1(Part I), 17 (2009)

Cummins, J.D.: Deregulating Property-Liability Insurance: Restoring Competition and Increasing Market Efficiency. AEI-Brookings Joint Center for Regulatory Studies, Washington, DC (2002)

Cummins, J.D.: Deregulating property-liability insurance. J. Risk Insur. 74(1), 269-272 (2007)

Cummins, J.D., Tennyson, S.: Controlling automobile insurance costs. J. Econ. Perspect. 6(2), 95-115 (1992)

Cuocci, V.V.: Dall'Assicurazione obbligatoria R.C. Auto alla no-fault insurance. Uno studio comparativo dei sistemi di traffic accident compensation. Università di Foggia Dipartimento di Giurisprudenza. Giuffrè Editore, pp. 1-278 (2013). ISBN: 8814180431

Dahlby, B.G.: Adverse selection and statistical discrimination. J. Public Econ. 20, 121-130 (1983)

De Santis, S.: Impact of Direct on RCA. Ania, Rome (2006)

Fersini, P., Melisi, G.: Stochastic model to evaluate the fair value of motor third-party liability under the direct reimbursement scheme and quantification of the capital requirement in a Solvency II perspective. Insur. Math. Econ. 68, 27-44 (2016). https://doi.org/10.1016/j.insmatheco.2016.02.002. ISSN: 0167-6687

Fersini, P., Crenca, C., Olivieri, G., Melisi, G., Forte, S.: Analisi del mercato assicurativo R.C. Auto per il Settore V-MOTOVEICOLI: distorsioni di pricing a seguito dell'introduzione del sistema di Indennizzo Diretto. Luiss University Press (2017). ISBN: 978-88-6856-076-8 [Analysis of the MTPL insurance market for motor vehicles, business line V-MOTORVEHICLE: pricing distortions with the introduction of the direct reimbursement system] 
Galli, G., Savino, C., 2007. Direct Reimbursement in Motor Liability Insurance. GIIA Volume LXX, Rome

Gastel, R.: Compulsory Auto Insurance. Insurance Issues Update. Insurance Information Institute, New York (1998)

Grabowski, H., Kip Viscusi, W., Evans, W.N.: Price and availability tradeoffs of automobile insurance regulation. J. Risk Insur. 56, 275-299 (1989)

Grace, M.F., Leverty, J.T.: How Tort reform affects insurance markets. J. Law Econ. Organ. 29, 12531278 (2012)

Graham, L., Xie, X.: The United States insurance market: characteristics and trends. In: Cummins J.D., Venard B. (eds) Handbook of International Insurance. Huebner International Series on Risk, Insurance and Economic Security, vol 26. Springer, Boston (2007). https://doi.org/10.1007/978-0-38734163-7

Insurance Europe: European Insurance-key fact. https:/www.insuranceeurope.eu/sites/default/files/ attachments/European\%20Insurance\%20-\%20Key\%20Facts\%20-\%20August\%202016.pdf (2016). Accessed 14 June 2018

Joost, R.H.: Automobile Insurance and No-Fault Law, 2nd edn. Lawyers Co-operative Pub. Co., Rochester (2002)

Kelly, M., Kleffner, A.E., Tomlinson, M.: First-party versus third party compensation for automobile accidents: evidence from Canada. Risk Manag. Insur. Rev. 13(1), 21-44 (2010)

National Association of Insurance Commissioners (NAIC): State Insurance Regulation: Key Facts and Market Trends. http://www.naic.org/state_report_cards/report_card_la.pdf (2016). Accessed 25 Jan 2018

Schermer, I.E., Schermer, W.J.: Automobile Liability Insurance, vol. 1, 4th edn. Thomson/West, Eagan (2004)

Schmitt, K.E.: European auto insurance pricing considerations. In: Casualty Actuarial Society Forum, Winter, pp. 141-158 (2000). Available at http://citeseerx.ist.psu.edu/viewdoc/download?doi=10.1.1 $.376 .3569 \&$ rep $=$ rep $1 \&$ type $=$ pdf\#page $=147$

Sugarman, S.D.: The Uninsured Motorist Puzzle, Crossroads: The Auto Accident Project, University of Wisconsin-Milwaukee, Special Edition, pp. 1-12 (1998)

Tennyson, S.: The impact of rate regulation on state automobile insurance markets. J. Insur. Regul. 15, 502-523 (1997)

Yu-Luen, M., Schmit, J.T.: Factors affecting the relative incidence of uninsured motorists claims. J. Risk Insur. 67, 281-294 (2000)

Publisher's Note Springer Nature remains neutral with regard to jurisdictional claims in published maps and institutional affiliations.

\section{Affiliations}

\section{Paola Fersini $^{1}$ (D) $\cdot$ Salvatore Forte ${ }^{2} \cdot$ Giuseppe Melisi $^{3} \cdot$ Gennaro Olivieri $^{1}$}

Salvatore Forte

s.forte@unifortunato.eu

Giuseppe Melisi

gmelisi@luiss.it

Gennaro Olivieri

olivieri@luiss.it

1 Department of Business and Management, Libera Università Internazionale degli Studi Sociali Guido Carli, Rome, Italy

2 Department of Law, Università Telematica Giustino Fortunato, Benevento, Italy

3 Department of Law, Economics and Quantitative Methods, Università degli Studi del Sannio, Benevento, Italy 